\title{
Recursive Minimum-Variance Filter Design for State-Saturated Complex Networks with Uncertain Coupling Strengths Subject to Deception Attacks
}

\author{
Hongyu Gao, Hongli Dong, Zidong Wang and Fei Han
}

\begin{abstract}
In this paper, the recursive filtering problem is investigated for state-saturated complex networks (CNs) subject to uncertain coupling strengths (UCSs) and deception attacks. The measurement signals transmitted via the communication network may suffer from deception attacks which are governed by Bernoulli-distributed random variables. The purpose of the problem under consideration is to design a minimum-variance filter for CNs with deception attacks, state saturations and UCSs such that upper bounds on the resulting error covariances are guaranteed. Then, the expected filter gains are acquired via minimizing the traces of such upper bounds and sufficient conditions are established to ensure the exponential mean-square boundedness of the filtering errors. At last, two simulation examples (including a practical application) are exploited to validate the effectiveness of our designed approach.
\end{abstract}

Index Terms-Complex networks, recursive filtering, state saturations, coupling uncertainties, deception attacks.

\section{INTRODUCTION}

The past decades have seen a recurring research interest in complex networks (CNs) because of their wide applications in a variety of practical situations such as economics, biology, and social science [29]-[31], [33], [36], [38], [39], [41], [47]. Basically, for many $\mathrm{CN}$ applications, it is indispensable for the network states to be utilized to fulfil the requirements for monitoring, approximation and optimization. However, due to the inherent characteristics (e.g. complicated structure and local interaction) of CNs, the network states are usually unavailable to the end user but only the measurement outputs can be accessible. As such, much research enthusiasm has recently been generated towards the state estimation problems for CNs.

Up to now, plenty of filtering/estimation algorithms have been devised for a great variety of CNs (see [5], [18], [27], [45], [48], [50]) with tremendous attention from both academia

This work was supported in part by the Engineering and Physical Sciences Research Council (EPSRC) of the UK, the Royal Society of the UK, and the Alexander von Humboldt Foundation of Germany.

$\mathrm{H}$. Gao is with the School of Electrical and Information Engineering, Northeast Petroleum University, Daqing 163318, China, and also with the Heilongjiang Provincial Key Laboratory of Networking and Intelligent Control, Northeast Petroleum University, Daqing 163318, China.

H. Dong and F. Han are with the Institute of Complex Systems and Advanced Control, Northeast Petroleum University, Daqing 163318, China, and also with the Heilongjiang Provincial Key Laboratory of Networking and Intelligent Control, Northeast Petroleum University, Daqing 163318, China (Email: shiningdhlevip.126.com)

Z. Wang is with the Department of Computer Science, Brunel University London, Uxbridge, Middlesex, UB8 3PH, United Kingdom. (Email: Zidong.Wang@brunel.ac.uk) and industry. In practical engineering, many complex systems have time-varying parameters that might be caused by different reasons, e.g. operating point shifting and parameter fluctuation [1], [2]. To address the filtering issues of time-varying CNs, various methods have been devised with examples including the finite-horizon $H_{\infty}$ filtering and recursive filtering (RF) algorithms, where the latter is most widely studied algorithm that has gained a great deal of research interest [5], [10], [15], [20]-[24].

In $\mathrm{CN}$ applications, an underlying assumption is that the coupling strengths among $\mathrm{CN}$ nodes can be described as certain known constants, see e.g. [30], [35], [40], [45]. This assumption is, unfortunately, quite restrictive in practice. For instance, it has been mentioned in [44] that coupling strengths among $\mathrm{CN}$ nodes might fluctuate due to various reasons such as noise disturbance and signal transmission congestion. Thus, the analysis/synthesis problems of CNs with uncertain coupling strengths (UCSs) have stirred certain research attention with preliminary results in [14], [26]. In [14], an adaptive scheme has been put forward to handle synchronization problems for $\mathrm{CNs}$ against the network deterioration caused by coupling uncertainties. In [26], some adaptive controllers have been designed for CNs with uncertain coupling matrices.

State saturation has been well recognized as a special kind of nonlinear constraints whose pervasive existence is largely due to the physical limits of the internal states of CNs. In fact, in the CN-related filtering problem, the presence of state saturations has a major impact on the filter performance since the state estimate is saturated. The state saturation phenomenon, if not adequately handled, may lead to performance degradation or even filter instability. In recent years, some initial works have been acquired on filtering problems of state-saturated CNs, see e.g. [8], [19]. For instance, an $H_{\infty}$ filter has been devised in [19] for state-saturated CNs with distributed delays and quantized measurements and under the event-triggering mechanism. Nevertheless, for time-varying state-saturated CNs with UCSs, relevant results on their RF problems are still scarce.

Along with the prevalence of utilizing industry networks, cyber-security issues have gradually become major concerns as open and unprotected communication networks are vulnerable to cyber attacks launched by adversaries. Recently, the security RF problems under cyber attacks have received much attention with many results available in the literature [4], [7], [17]. For example, the distributed RF method has been proposed in [6] for time-delayed stochastic systems with deception attacks 
This article has been accepted for publication in a future issue of this journal, but has not been fully edited. Content may change prior to final publication. Citation information: DOI 10.1109/TCYB.2021.3067822, IEEE Transactions on Cybernetics

and quantization effects. The variance-constrained distributed filtering issue has been solved in [32] for sensor networks under deception attacks. In [43], a filter has been designed for nonlinear systems with time-delays, where both stochastic deception attacks and sensor saturations have been taken into account. However, to date, RF issues for $\mathrm{CNs}$ with state saturations and UCSs subject to deception attacks have not been thoroughly discussed yet.

Concluding the discussions made thus far, it is both practically and theoretically significant to cope with the RF issue for state-saturated CNs with UCSs under deception attacks. In order to handle this issue, we are confronted with three main difficulties: 1) how to establish an appropriate state-saturated $\mathrm{CN}$ model with coupling uncertainties; 2) how to devise a suitable filter for the concerned $\mathrm{CNs}$; 3) how to guarantee the exponential mean-square boundedness (EMSB) of the filtering errors. Accordingly, our primary contributions are: 1) a novel CN model is proposed to tackle the concurrence of state saturations, UCSs, and deception attacks; 2) upper bounds on error covariances are obtained with filter parameters calculated by utilizing both local and neighboring information; and 3) sufficient conditions are presented to guarantee the EMSB of filtering errors.

\section{Problem Formulation and Preliminaries}

Consider a state-saturated $\mathrm{CN}$ with UCSs as follows:

$$
\left\{\begin{aligned}
x_{i}^{k+1} & =\sigma_{i}\left(f\left(x_{i}^{k}\right)+\sum_{j=1}^{N}\left(\omega_{i j}+\Delta \omega_{i j}\right) \Gamma x_{j}^{k}\right)+D_{i}^{k} \varpi_{i}^{k} \\
y_{i}^{k} & =G_{i}^{k} x_{i}^{k}+v_{i}^{k}
\end{aligned}\right.
$$

where $x_{i}^{k} \in \mathbb{R}^{n}(i=1,2, \ldots, N)$ is the state of the $i$ th node and $y_{i}^{k} \in \mathbb{R}^{m}$ is the associated measurement output. $\Gamma \triangleq \operatorname{diag}\left\{b_{1}, b_{2}, \cdots, b_{n}\right\}>0$ denotes the inner-coupling matrix where $b_{s} \neq 0(s=1,2, \ldots, n)$ is the coupling strength. $f(\cdot)$ denotes a known nonlinear function. $\Omega \triangleq\left[\omega_{i j}\right](j=$ $1,2, \ldots, N)$ is the certain coupling strength coefficient where $\omega_{i j} \geq 0 . \Delta \omega_{i j}$ is the UCS with $\left|\Delta \omega_{i j}\right| \leq \varsigma_{j}(j=1, \cdots, N)$. The zero-mean white Gaussian noises $\varpi_{i}^{k} \in \mathbb{R}^{r_{1}}$ and $v_{i}^{k} \in \mathbb{R}^{r_{2}}$ have covariances $R_{i \varpi}^{k}$ and $R_{i v}^{k}$, respectively. Assume that $\varpi_{i}^{k}$ and $v_{i}^{k}$ are mutually uncorrelated for any $i$ and $k . D_{i}^{k}$ and $G_{i}^{k}$ ( $G_{i}^{k}$ is invertible) are known time-varying system matrices.

The saturation function $\sigma(\cdot): \mathbb{R}^{n} \mapsto \mathbb{R}^{n}$ is defined as

$$
\sigma_{i}(\pi) \triangleq\left[\begin{array}{llll}
\sigma_{i 1}\left(\pi_{i 1}\right) & \sigma_{i 2}\left(\pi_{i 2}\right) & \cdots & \sigma_{i n}\left(\pi_{i n}\right)
\end{array}\right]^{T}
$$

where

$$
\begin{aligned}
\pi & \triangleq\left[\begin{array}{llll}
\pi_{i 1} & \pi_{i 2} & \cdots & \pi_{i n}
\end{array}\right]^{T}, \\
\sigma_{i s}\left(\pi_{i s}\right) & \triangleq \operatorname{sgn}\left(\pi_{i s}\right) \min \left\{\pi_{i s}^{\max },\left|\pi_{i s}\right|\right\}, s=1,2, \ldots, n
\end{aligned}
$$

in which $\operatorname{sgn}(\cdot)$ denotes a signum function, and $\pi_{i s}^{\max }$ is the $s$ th element of the vector $\pi_{i}^{\max }$ representing the saturation level vector.

Let us now introduce the transmission model under deception attacks. In general, the successes of deception attacks launched by the adversaries are dependent on the network conditions and the performance of the protection equipment.
Therefore, the deception attacks can be considered as randomly occurring from the defenders' perspective and the transmission signals subject to deception attacks can then be modeled as follows:

$$
\left\{\begin{array}{l}
\tilde{y}_{i}^{k}=y_{i}^{k}+\alpha_{i}^{k} \zeta_{i}^{k} \\
\zeta_{i}^{k}=-y_{i}^{k}+\xi_{k}
\end{array}\right.
$$

where $\tilde{y}_{i}^{k}$ is the received signal of the $i$-th node from adjacent node, and $\xi_{k} \in \mathbb{R}^{m}$ represents the non-zero signal sent by adversaries satisfying $\left\|\xi_{k}\right\| \leq \theta$ for an arbitrary given positive scalar $\theta$.

The stochastic variable $\alpha_{i}^{k}$ is Bernoulli-distributed with the following probabilities:

$$
\operatorname{Prob}\left\{\alpha_{i}^{k}=0\right\}=1-\bar{\alpha}_{i}, \quad \operatorname{Prob}\left\{\alpha_{i}^{k}=1\right\}=\bar{\alpha}_{i}
$$

where $\bar{\alpha}_{i} \in[0,1)$ is a known constant.

Letting $\hat{x}_{i}^{k+1 \mid k}$ and $\hat{x}_{i}^{k+1 \mid k+1}$ represent, respectively, the predicted and estimated values of $x_{i}^{k+1}$, we put forward the following filter:

$$
\left\{\begin{aligned}
\hat{x}_{i}^{k+1 \mid k} & =\sigma_{i}\left(f_{i}\left(\hat{x}_{i}^{k \mid k}\right)+\sum_{j=1}^{N} \omega_{i j} \Gamma \hat{x}_{j}^{k \mid k}\right) \\
\hat{x}_{i}^{k+1 \mid k+1} & =\hat{x}_{i}^{k+1 \mid k}+K_{i}^{k+1}\left(\tilde{y}_{i}^{k+1}-G_{i}^{k+1} \hat{x}_{i}^{k+1 \mid k}\right)
\end{aligned}\right.
$$

where $K_{i}^{k+1}$ is the gain to be determined.

In order to facilitate our analysis, we denote

$$
\begin{aligned}
e_{i}^{k+1 \mid k} & \triangleq x_{i}^{k+1}-\hat{x}_{i}^{k+1 \mid k}, \\
e_{i}^{k+1 \mid k+1} & \triangleq x_{i}^{k+1}-\hat{x}_{i}^{k+1 \mid k+1}, \\
P_{i}^{k+1 \mid k} & \triangleq \mathbb{E}\left\{e_{i}^{k+1 \mid k}\left(e_{i}^{k+1 \mid k}\right)^{T}\right\}, \\
P_{i}^{k+1 \mid k+1} & \triangleq \mathbb{E}\left\{e_{i}^{k+1 \mid k+1}\left(e_{i}^{k+1 \mid k+1}\right)^{T}\right\} .
\end{aligned}
$$

The main objectives of this paper are to: 1) obtain upper bounds on $P_{i}^{k+1 \mid k+1}$;2) calculate the time-varying filter gain through minimizing the trace of the obtained bounds; and 3) discuss the EMSB of the filtering errors.

\section{MAin RESUlts}

In this section, we will design a RF method to estimate states of the state-saturated CNs with UCSs subject to deception attacks. First, we calculate upper bounds on $P_{i}^{k+1 \mid k+1}$. Then, filter gains $K_{i}^{k+1}$ are obtained by minimizing the trace of the acquired upper bound. Finally, the EMSB of the filtering errors are discussed.

Before proceeding, we need the following lemmas.

Lemma 1: [24] For vectors $a, b \in \mathbb{R}^{n}$, the inequality

$$
a b^{T}+b a^{T} \leq \lambda a a^{T}+\lambda^{-1} b b^{T}
$$

holds where $\lambda>0$ is a positive scalar.

Lemma 2: [8] For any $x_{1}, x_{2} \in \mathbb{R}$, there exists $\varepsilon_{h} \in[0,1]$ such that

$$
\sigma_{h}\left(x_{1}\right)-\sigma_{h}\left(x_{2}\right)=\varepsilon_{h}\left(x_{1}-x_{2}\right), \quad h=1,2, \ldots, n
$$

where $\sigma_{h}(\cdot)$ is the saturation function defined in (2)-(4). 
This article has been accepted for publication in a future issue of this journal, but has not been fully edited. Content may change prior to final publication. Citation information: DOI 10.1109/TCYB.2021.3067822, IEEE Transactions on Cybernetics

Lemma 3: [34] For matrices $\mathscr{P}, \mathscr{Q}, \mathscr{R}$ and $\mathscr{S}$, symmetric matrix $Z>0$ and constant $\epsilon>0$ satisfying $\mathscr{R} \mathscr{R}^{T}<I$ and $\epsilon^{-1} I-\mathscr{S} Z \mathscr{S}^{T}>0$, the inequality

$$
\begin{aligned}
& (\mathscr{P}+\mathscr{Q} \mathscr{R} \mathscr{S}) Z(\mathscr{P}+\mathscr{Q} \mathscr{R} \mathscr{S})^{T} \\
\leq & \mathscr{P}\left(Z^{-1}-\epsilon \mathscr{S}^{T} \mathscr{S}\right)^{-1} \mathscr{P}^{T}+\epsilon^{-1} \mathscr{Q} \mathscr{Q}^{T}
\end{aligned}
$$

holds.

Lemma 4: [42] For $0 \leq k \leq n$, suppose that $X=X^{T}>$ $0, \mathcal{M}_{k}(X)=\mathcal{M}_{k}^{T}(X) \in \mathbb{R}^{L \times L}$ and $\mathcal{N}_{k}(X)=\mathcal{N}_{k}^{T}(X) \in$ $\mathbb{R}^{L \times L}$. If there exists $\mathcal{Z}=\mathcal{Z}^{T}>X$ such that

$$
\mathcal{M}_{k}(X) \geq \mathcal{M}_{k}(\mathcal{Z}), \quad \mathcal{N}_{k}(X) \geq \mathcal{M}_{k}(X),
$$

then solutions $\mathcal{R}_{k}$ and $\mathcal{S}_{k}$ to

$$
\mathcal{R}_{k}=\mathcal{M}_{k}\left(\mathcal{R}_{k-1}\right), \mathcal{S}_{k}=\mathcal{N}_{k}\left(\mathcal{S}_{k-1}\right), \mathcal{R}_{0}=\mathcal{S}_{0}>0
$$

satisfy $\mathcal{R}_{k} \leq \mathcal{S}_{k}$.

Lemma 5: [28] Given constant matrices $\mathcal{T}_{1}, \mathcal{T}_{2}$ and $\mathcal{T}_{3}$ where $0<\mathcal{T}_{1}=\mathcal{T}_{1}^{T}$ and $0<\mathcal{T}_{2}=\mathcal{T}_{2}^{T}$, then $\mathcal{T}_{1}-\mathcal{T}_{3}^{T} \mathcal{T}_{2} \mathcal{T}_{3} \geq$ 0 if and only if

$$
\begin{aligned}
& {\left[\begin{array}{cc}
\mathcal{T}_{1} & \mathcal{T}_{3}^{T} \\
\mathcal{T}_{3} & \mathcal{T}_{2}^{-1}
\end{array}\right] \geq 0, \quad \text { or } \quad\left[\begin{array}{cc}
\mathcal{T}_{2}^{-1} & \mathcal{T}_{3} \\
\mathcal{T}_{3}^{T} & \mathcal{T}_{1}
\end{array}\right] \geq 0,} \\
& \text { or } \mathcal{T}_{2}^{-1}-\mathcal{T}_{3} \mathcal{T}_{1}^{-1} \mathcal{T}_{3}^{T} \geq 0 .
\end{aligned}
$$

Lemma 6: [37] For any stochastic process $V_{k}\left(\vartheta_{k}\right)$ and real number $\delta_{\min }, \delta_{\max }, u>0$ and $0<\beta \leq 1$, if

$$
\delta_{\min }\left\|\vartheta_{k}\right\|^{2} \leq V_{k}\left(\vartheta_{k}\right) \leq \delta_{\max }\left\|\vartheta_{k}\right\|^{2}
$$

and

$$
\mathbb{E}\left\{V_{k}\left(\vartheta_{k}\right) \mid \vartheta_{k-1}\right\} \leq(1-\beta) V_{k-1}\left(\vartheta_{k-1}\right)+u,
$$

then the EMSB of $\vartheta_{k}$ is ensured , i.e.,

$$
\mathbb{E}\left\{\left\|\vartheta_{k}\right\|^{2}\right\} \leq \frac{\delta_{\max }}{\delta_{\min }} \mathbb{E}\left\{\left\|\vartheta_{0}\right\|^{2}\right\}(1-\beta)^{k}+\frac{u}{\delta_{\min }} \sum_{i=1}^{k}(1-\beta)^{i} .
$$

Theorem 1: The following recursions hold:

$$
\begin{aligned}
& P_{i}^{k+1 \mid k} \\
= & D_{i}^{k} R_{i \varpi}^{k}\left(D_{i}^{k}\right)^{T}+\mathbb{E}\left\{\Theta _ { i } ^ { k } ( F _ { i } ^ { k } + M _ { i } ^ { k } W _ { i } ^ { k } ) P _ { i } ^ { k | k } \left(F_{i}^{k}\right.\right. \\
& \left.\left.+M_{i}^{k} W_{i}^{k}\right)^{T} \Theta_{i, k}^{T}\right\}+\sum_{j=1}^{N} \omega_{i j} \mathbb{E}\left\{\Theta _ { i } ^ { k } \left(\left(F_{i}^{k}+M_{i}^{k} W_{i}^{k}\right) e_{i}^{k \mid k}\right.\right. \\
& \left.\left.\times\left(e_{j}^{k \mid k}\right)^{T} \Gamma^{T}+\Gamma e_{j}^{k \mid k}\left(e_{i}^{k \mid k}\right)^{T}\left(F_{i}^{k}+M_{i}^{k} W_{i}^{k}\right)^{T}\right)\left(\Theta_{i}^{k}\right)^{T}\right\} \\
& +\sum_{j=1}^{N} \Delta \omega_{i j} \mathbb{E}\left\{\Theta _ { i } ^ { k } \left(\Gamma x_{j}^{k}\left(e_{i}^{k \mid k}\right)^{T}\left(F_{i}^{k}+M_{i}^{k} W_{i}^{k}\right)^{T}\right.\right. \\
& \left.\left.+\left(F_{i}^{k}+M_{i}^{k} W_{i}^{k}\right) e_{i}^{k \mid k}\left(x_{j}^{k}\right)^{T} \Gamma^{T}\right)\left(\Theta_{i}^{k}\right)^{T}\right\}+\sum_{j=1}^{N} \sum_{p=1}^{N} \Delta \omega_{i j} \\
& \times \Delta \omega_{i p} \mathbb{E}\left\{\Theta_{i}^{k} \Gamma x_{j}^{k}\left(x_{p}^{k}\right)^{T} \Gamma^{T}\left(\Theta_{i}^{k}\right)^{T}\right\}+\sum_{j=1}^{N} \sum_{p=1}^{N} \omega_{i j} \omega_{i p} \\
& \times \mathbb{E}\left\{\Theta_{i}^{k} \Gamma e_{p}^{k \mid k}\left(e_{j}^{k \mid k}\right)^{T} \Gamma^{T}\left(\Theta_{i}^{k}\right)^{T}\right\}+\sum_{j=1}^{N} \sum_{p=1}^{N} \Delta \omega_{i j} \omega_{i p} \\
& \times \mathbb{E}\left\{\Theta_{i}^{k} \Gamma\left(x_{j}^{k}\left(e_{p}^{k \mid k}\right)^{T}+e_{p}^{k \mid k} x_{j, k}^{T}\right) \Gamma^{T}\left(\Theta_{i}^{k}\right)^{T}\right\}
\end{aligned}
$$

and

$$
\begin{aligned}
& P_{i}^{k+1 \mid k+1} \\
= & \left(I-K_{i}^{k+1} G_{i}^{k+1}\right) P_{i}^{k+1 \mid k}\left(I-K_{i}^{k+1} G_{i}^{k+1}\right)^{T} \\
& +\left(1-\bar{\alpha}_{i}\right) K_{i}^{k+1} R_{i v}^{k+1}\left(K_{i}^{k+1}\right)^{T}+\mathbb{E}\left\{\bar{\alpha}_{i} K_{i}^{k+1} \xi_{k+1} \xi_{k+1}^{T}\right. \\
& \times\left(K_{i}^{k+1}\right)^{T}+\bar{\alpha}_{i} K_{i}^{k+1} G_{i}^{k+1} x_{i}^{k+1}\left(x_{i}^{k+1}\right)^{T}\left(G_{i}^{k+1}\right)^{T}\left(K_{i}^{k+1}\right)^{T} \\
& +\bar{\alpha}_{i}\left(I-K_{i}^{k+1} G_{i}^{k+1}\right) e_{i}^{k+1 \mid k}\left(x_{i}^{k+1}\right)^{T}\left(G_{i}^{k+1}\right)^{T}\left(K_{i}^{k+1}\right)^{T} \\
& -\bar{\alpha}_{i} K_{i}^{k+1} G_{i}^{k+1} x_{i}^{k+1} \xi_{k+1}^{T}\left(K_{i}^{k+1}\right)^{T}+\bar{\alpha}_{i} K_{i}^{k+1} G_{i}^{k+1} \\
& \times x_{i}^{k+1}\left(e_{i}^{k+1 \mid k}\right)^{T}\left(I-K_{i}^{k+1} G_{i}^{k+1}\right)^{T}-\bar{\alpha}_{i} K_{i}^{k+1} \xi_{k+1} \\
& \times\left(x_{i}^{k+1}\right)^{T}\left(G_{i}^{k+1}\right)^{T}\left(K_{i}^{k+1}\right)^{T}-\bar{\alpha}_{i}\left(I-K_{i}^{k+1} G_{i}^{k+1}\right) \\
& \times e_{i}^{k+1 \mid k} \xi_{k+1}^{T}\left(K_{i}^{k+1}\right)^{T}-\bar{\alpha}_{i} K_{i}^{k+1} \xi_{k+1}\left(e_{i}^{k+1 \mid k}\right)^{T} \\
& \left.\times\left(I-K_{i}^{k+1} G_{i}^{k+1}\right)^{T}\right\}
\end{aligned}
$$

where $\Theta_{i}^{k} \triangleq \operatorname{diag}\left\{\left(\varepsilon_{i}^{k}\right)^{(1)},\left(\varepsilon_{i}^{k}\right)^{(2)}, \cdots,\left(\varepsilon_{i}^{k}\right)^{(n)}\right\},\left(\varepsilon_{i}^{k}\right)^{(\nu)} \in$ $[0,1](\nu=1,2, \ldots, n)$ and $\left.F_{i}^{k} \triangleq \frac{\partial f\left(x_{i}^{k}\right)}{\partial x_{i}^{k}}\right|_{x_{i}^{k}=\hat{x}_{i}^{k \mid k}}$. The scaling matrix $M_{i}^{k}$ is problem-dependent and the unknown matrix $W_{i}^{k}$ stands for linearization errors and satisfies $W_{i}^{k}\left(W_{i}^{k}\right)^{T} \leq I$.

Proof: It follows from Lemma 1 and (1) that

$$
\begin{aligned}
e_{i}^{k+1 \mid k}= & x_{i}^{k+1}-\hat{x}_{i}^{k+1 \mid k} \\
= & \sigma_{i}\left(f_{i}\left(x_{i}^{k}\right)+\sum_{j=1}^{N}\left(\omega_{i j}+\Delta \omega_{i j}\right) \Gamma x_{j}^{k}\right) \\
& -\sigma_{i}\left(f_{i}\left(\hat{x}_{i}^{k \mid k}\right)+\sum_{j=1}^{N} \omega_{i j} \Gamma \hat{x}_{j}^{k \mid k}\right)+D_{i}^{k} \varpi_{i}^{k} \\
= & \Theta_{i}^{k}\left(f_{i}\left(x_{i}^{k}\right)-f_{i}\left(\hat{x}_{i}^{k \mid k}\right)+\sum_{j=1}^{N} \Delta \omega_{i j} \Gamma x_{j}^{k}\right. \\
& \left.+\sum_{j=1}^{N} \omega_{i j} \Gamma e_{j}^{k \mid k}\right)+D_{i}^{k} \varpi_{i}^{k} .
\end{aligned}
$$

Expanding $f\left(x_{i}^{k}\right)$ around $\hat{x}_{i}^{k \mid k}$ generates

$$
f\left(x_{i}^{k}\right)=f\left(\hat{x}_{i}^{k \mid k}\right)+F_{i}^{k} e_{i}^{k \mid k}+o\left(\left|e_{i}^{k \mid k}\right|\right)
$$

where

$$
\left.F_{i}^{k} \triangleq \frac{\partial f\left(x_{i}^{k}\right)}{\partial x_{i}^{k}}\right|_{x_{i}^{k}=\hat{x}_{i}^{k \mid k}}
$$

is the Jacobian matrix. $o\left(\left|e_{i}^{k \mid k}\right|\right)$ is the high-order term of Taylor series expression. According to [15], $o\left(\left|e_{i}^{k \mid k}\right|\right)$ is rewritten as

$$
o\left(\left|e_{i}^{k \mid k}\right|\right) \triangleq M_{i}^{k} W_{i}^{k} e_{i}^{k \mid k} .
$$

Substituting (20)-(21) into (19), we obtain

$$
\begin{aligned}
e_{i}^{k+1 \mid k}= & x_{i}^{k+1}-\hat{x}_{i}^{k+1 \mid k} \\
= & \Theta_{i}^{k}\left(F_{i}^{k}+M_{i}^{k} W_{i}^{k}\right) e_{i}^{k \mid k}+\Theta_{i}^{k} \sum_{j=1}^{N} \Delta \omega_{i j} \Gamma x_{j}^{k} \\
& +\Theta_{i}^{k} \sum_{j=1}^{N} \omega_{i j} \Gamma e_{j}^{k \mid k}+D_{i}^{k} \varpi_{i}^{k} .
\end{aligned}
$$

On account of $P_{i}^{k+1 \mid k} \triangleq \mathbb{E}\left\{e_{i}^{k+1 \mid k}\left(e_{i}^{k+1 \mid k}\right)^{T}\right\}$, one has $P_{i}^{k+1 \mid k}$ 
This article has been accepted for publication in a future issue of this journal, but has not been fully edited. Content may change prior to final publication. Citation information: DOI 10.1109/TCYB.2021.3067822, IEEE Transactions on Cybernetics

$$
\begin{aligned}
= & \mathbb{E}\left\{\left(\Theta_{i}^{k}\left(F_{i}^{k}+M_{i}^{k} W_{i}^{k}\right) e_{i}^{k \mid k}+\Theta_{i}^{k} \sum_{j=1}^{N} \Delta \omega_{i j} \Gamma x_{j}^{k}\right.\right. \\
& \left.+\Theta_{i}^{k} \sum_{j=1}^{N} \omega_{i j} \Gamma e_{j}^{k \mid k}+D_{i}^{k} \varpi_{i}^{k}\right)\left(\Theta_{i}^{k}\left(F_{i}^{k}+M_{i}^{k} W_{i}^{k}\right)\right. \\
& \times e_{i}^{k \mid k}+\Theta_{i}^{k} \sum_{j=1}^{N} \Delta \omega_{i j} \Gamma x_{j}^{k}+\Theta_{i}^{k} \sum_{j=1}^{N} \omega_{i j} \Gamma e_{j}^{k \mid k} \\
& \left.\left.+D_{i}^{k} \varpi_{i}^{k}\right)^{T}\right\} .
\end{aligned}
$$

Thus, it is easy to obtain (17) from (23).

From (1), (5) and (7), one has

$$
\begin{aligned}
e_{i}^{k+1 \mid k+1}= & x_{i}^{k+1}-\hat{x}_{i}^{k+1 \mid k+1} \\
= & \left(I-K_{i}^{k+1} G_{i}^{k+1}\right) e_{i}^{k+1 \mid k}+\bar{\alpha}_{i} K_{i}^{k+1} \\
& \times\left(G_{i}^{k+1} x_{i}^{k+1}+v_{i}^{k+1}-\xi_{k+1}\right)+\tilde{\alpha}_{i}^{k+1} \\
& \times K_{i}^{k+1}\left(G_{i}^{k+1} x_{i}^{k+1}+v_{i}^{k+1}-\xi_{k+1}\right) \\
& -K_{i}^{k+1} v_{i}^{k+1}
\end{aligned}
$$

where $\tilde{\alpha}_{i}^{k+1} \triangleq \alpha_{i}^{k+1}-\bar{\alpha}_{i}$. Therefore, $P_{i}^{k+1 \mid k+1}$ is derived as

$$
\begin{aligned}
& P_{i}^{k+1 \mid k+1} \\
= & \left(I-K_{i}^{k+1} G_{i}^{k+1}\right) P_{i}^{k+1 \mid k}\left(I-K_{i}^{k+1} G_{i}^{k+1}\right)^{T} \\
& +\mathbb{E}\left\{\bar{\alpha}_{i}\left(I-K_{i}^{k+1} G_{i}^{k+1}\right) e_{i}^{k+1 \mid k}\left(x_{i}^{k+1}\right)^{T}\left(G_{i}^{k+1}\right)^{T}\left(K_{i}^{k+1}\right)^{T}\right. \\
& -\bar{\alpha}_{i}\left(I-K_{i}^{k+1} G_{i}^{k+1}\right) e_{i}^{k+1 \mid k} \xi_{k+1}^{T}\left(K_{i}^{k+1}\right)^{T} \\
& +\bar{\alpha}_{i}^{2} K_{i}^{k+1} G_{i}^{k+1} x_{i}^{k+1}\left(x_{i}^{k+1}\right)^{T}\left(G_{i}^{k+1}\right)^{T}\left(K_{i}^{k+1}\right)^{T} \\
& +\bar{\alpha}_{i} K_{i}^{k+1} G_{i}^{k+1} x_{i}^{k+1}\left(e_{i}^{k+1 \mid k}\right)^{T}\left(I-K_{i}^{k+1} G_{i}^{k+1}\right)^{T} \\
& -\bar{\alpha}_{i}^{2} K_{i}^{k+1} G_{i}^{k+1} x_{i}^{k+1} \xi_{k+1}^{T}\left(K_{i}^{k+1}\right)^{T}+\left(\bar{\alpha}_{i}-1\right)^{2} \\
& \times K_{i}^{k+1} R_{i v}^{k+1}\left(K_{i}^{k+1}\right)^{T}+\bar{\alpha}_{i}^{2} K_{i}^{k+1} \xi_{k+1} \xi_{k+1}^{T}\left(K_{i}^{k+1}\right)^{T} \\
& -\bar{\alpha}_{i} K_{i}^{k+1} \xi_{k+1}\left(e_{i}^{k+1 \mid k}\right)^{T}\left(I-K_{i}^{k+1} G_{i}^{k+1}\right)^{T} \\
& -\bar{\alpha}_{i}^{2} K_{i}^{k+1} \xi_{k+1}\left(x_{i}^{k+1}\right)^{T}\left(G_{i}^{k+1}\right)^{T}\left(K_{i}^{k+1}\right)^{T}+\bar{\alpha}_{i}\left(1-\bar{\alpha}_{i}\right) \\
& \times\left(K_{i}^{k+1} G_{i}^{k+1} x_{i}^{k+1}\left(x_{i}^{k+1}\right)^{T}\left(G_{i}^{k+1}\right)^{T}\left(K_{i}^{k+1}\right)^{T}\right. \\
& +K_{i}^{k+1} R_{i v}^{k+1}\left(K_{i}^{k+1}\right)^{T}+K_{i}^{k+1} \xi_{k+1} \xi_{k+1}^{T}\left(K_{i}^{k+1}\right)^{T} \\
& -K_{i}^{k+1} G_{i}^{k+1} x_{i}^{k+1} \xi_{k+1}^{T}\left(K_{i}^{k+1}\right)^{T} \\
& \left.\left.-K_{i}^{k+1} \xi_{k+1}\left(x_{i}^{k+1}\right)^{T}\left(G_{i}^{k+1}\right)^{T}\left(K_{i}^{k+1}\right)^{T}\right)\right\} .
\end{aligned}
$$

Remark 1: It is worth mentioning that some uncertain terms are included in (18) because of the consideration of the deception attacks and the noises, and this makes it impossible to accurately compute $P_{i}^{k+1 \mid k+1}$ and $K_{i}^{k+1}$. To deal with this problem, an alternative method for finding upper bounds on $P_{i}^{k+1 \mid k+1}$ is proposed through employing mathematical induction, and then the filter gain $K_{i}^{k+1}$ is obtained via minimizing traces of such upper bounds.

Theorem 2: Let model (1), positive scalars $\lambda_{1}, \rho_{1}, \rho_{2}, \rho_{3}$ and $\eta$ and initial conditions $P_{i}^{0 \mid 0} \leq \Phi_{i}^{0 \mid 0}$ be given. If

$$
\Phi_{i}^{k+1 \mid k}=a_{i}^{k} I+D_{i}^{k} R_{i \varpi}^{k}\left(D_{i}^{k}\right)^{T}
$$

and

$$
\Phi_{i}^{k+1 \mid k+1}
$$

$$
\begin{aligned}
= & \left(1+\bar{\alpha}_{i} \rho_{1}+\bar{\alpha}_{i} \rho_{3}\right)\left(I-K_{i}^{k+1} G_{i}^{k+1}\right) \Phi_{i}^{k+1 \mid k} \\
& \times\left(I-K_{i}^{k+1} G_{i}^{k+1}\right)^{T}+2 \bar{\alpha}_{i}\left(1+\rho_{1}^{-1}+\rho_{2}\right) K_{i}^{k+1} \\
& \times G_{i}^{k+1} \Phi_{i}^{k+1 \mid k}\left(G_{i}^{k+1}\right)^{T}\left(K_{i}^{k+1}\right)^{T}+2 \bar{\alpha}_{i}\left(1+\rho_{1}^{-1}+\rho_{2}\right) \\
& \times K_{i}^{k+1} G_{i}^{k+1} \hat{x}_{i}^{k+1 \mid k} \hat{x}_{i}^{T}(k+1 \mid k)\left(G_{i}^{k+1}\right)^{T}\left(K_{i}^{k+1}\right)^{T} \\
& +\bar{\alpha}_{i}\left(1+\rho_{2}^{-1}+\rho_{3}^{-1}\right) \theta^{2} K_{i}^{k+1}\left(K_{i}^{k+1}\right)^{T} \\
& +\left(1-\bar{\alpha}_{i}\right) K_{i}^{k+1} R_{i v}^{k+1}\left(K_{i}^{k+1}\right)^{T}
\end{aligned}
$$

admit positive-definite solutions such that, for all $k \geq 0$, the constraint $\eta^{-1} I<\Phi_{i}^{k \mid k}$ is satisfied, then matrices $\Phi_{i}^{k+1 \mid k}$ and $\Phi_{i}^{k+1 \mid k+1}$ are, respectively, the upper bounds on $P_{i}^{k+1 \mid k}$ and $P_{i}^{k+1 \mid k+1}$, i.e.,

$$
\begin{aligned}
P_{i}^{k+1 \mid k} & \leq \Phi_{i}^{k+1 \mid k}, \\
P_{i}^{k+1 \mid k+1} & \leq \Phi_{i}^{k+1 \mid k+1}
\end{aligned}
$$

where

$$
\begin{aligned}
a_{i}^{k} \triangleq & \min \left\{z_{i}^{k}, 4 \bar{\pi}_{i}\right\}, \\
z_{i}^{k} \triangleq & \left(1+\lambda_{1} \bar{\omega}_{i}+\bar{\varsigma}\right) \operatorname{tr}\left(F_{i}^{k}\left(\left(\Phi_{i}^{k \mid k}\right)^{-1}-\eta I\right)^{-1}\left(F_{i}^{k}\right)^{T}+\eta^{-1}\right. \\
& \left.\times M_{i}^{k}\left(M_{i}^{k}\right)^{T}\right)+\left(\lambda_{1}^{-1}+\bar{\varsigma}+\bar{\omega}_{i}\right) \sum_{j=1}^{N} \omega_{i j} \operatorname{tr}\left(\Gamma \Phi_{j}^{k \mid k} \Gamma^{T}\right) \\
& +2\left(1+\bar{\omega}_{i}+\bar{\varsigma}\right) \sum_{j=1}^{N} \varsigma_{j} \operatorname{tr}\left(\Gamma\left(\Phi_{j}^{k \mid k}+\hat{x}_{j}^{k \mid k}\left(\hat{x}_{j}^{k \mid k}\right)^{T}\right) \Gamma^{T}\right), \\
\bar{\pi}_{i} \triangleq & \sum_{h=1}^{n}\left(\pi_{i h}^{\max }\right)^{2}, \quad \bar{\omega}_{i} \triangleq \sum_{j=1}^{N} \omega_{i j}, \quad \bar{\varsigma} \triangleq \sum_{j=1}^{N} \varsigma_{j} .
\end{aligned}
$$

The gain matrix $K_{i}^{k+1}$ is calculated by

$$
K_{i}^{k+1}=\left(1+\bar{\alpha}_{i} \rho_{1}+\bar{\alpha}_{i} \rho_{3}\right) \Phi_{i}^{k+1 \mid k}\left(G_{i}^{k+1}\right)^{T}\left(\Pi_{i}^{k+1}\right)^{-1}
$$

where

$$
\begin{aligned}
& \Pi_{i}^{k+1} \\
\triangleq & G_{i}^{k+1}\left(\left(1+\bar{\alpha}_{i} \rho_{1}+\bar{\alpha}_{i} \rho_{3}\right) \Phi_{i}^{k+1 \mid k}+2 \bar{\alpha}_{i}\left(1+\rho_{1}^{-1}+\rho_{2}\right)\right. \\
& \left.\times\left(\Phi_{i}^{k+1 \mid k}+\hat{x}_{i}^{k+1 \mid k}\left(\hat{x}_{i}^{k+1 \mid k}\right)^{T}\right)\right)\left(G_{i}^{k+1}\right)^{T}+\bar{\alpha}_{i}\left(1+\rho_{2}^{-1}\right. \\
& \left.+\rho_{3}^{-1}\right) \theta^{2} I+\left(1-\bar{\alpha}_{i}\right) R_{i v}^{k+1} .
\end{aligned}
$$

Proof: The proof is carried out by resorting to mathematical induction. Assume that $P_{i}^{k \mid k} \leq \Phi_{i}^{k \mid k}$.

Observing the terms in (17), one knows from Lemmas 1-2 that

$$
\begin{aligned}
& \sum_{j=1}^{N} \omega_{i j} \mathbb{E}\left\{\Theta _ { i } ^ { k } \left(\left(F_{i}^{k}+M_{i}^{k} W_{i}^{k}\right) e_{i}^{k \mid k}\left(e_{j}^{k \mid k}\right)^{T} \Gamma^{T}\right.\right. \\
& \left.\left.+\Gamma e_{j}^{k \mid k}\left(e_{i}^{k \mid k}\right)^{T}\left(F_{i}^{k}+M_{i}^{k} W_{i}^{k}\right)^{T}\right)\left(\Theta_{i}^{k}\right)^{T}\right\} \\
\leq & \sum_{j=1}^{N} \omega_{i j}\left(\lambda_{1}\left(F_{i}^{k}+M_{i}^{k} W_{i}^{k}\right) P_{i}^{k \mid k}\left(F_{i}^{k}+M_{i}^{k} W_{i}^{k}\right)^{T}\right. \\
& \left.+\lambda_{1}^{-1} \Gamma P_{j}^{k \mid k} \Gamma^{T}\right)
\end{aligned}
$$

$$
\sum_{j=1}^{N} \Delta \omega_{i j} \mathbb{E}\left\{\Theta _ { i } ^ { k } \left(\Gamma x_{j}^{k}\left(e_{i}^{k \mid k}\right)^{T}\left(F_{i}^{k}+M_{i}^{k} W_{i}^{k}\right)^{T}\right.\right.
$$




$$
\begin{aligned}
& \left.\left.+\left(F_{i}^{k}+M_{i}^{k} W_{i}^{k}\right) e_{i}^{k \mid k}\left(x_{j}^{k}\right)^{T} \Gamma^{T}\right)\left(\Theta_{i}^{k}\right)^{T}\right\} \\
\leq & \sum_{j=1}^{N} \varsigma_{j}\left(\left(F_{i}^{k}+M_{i}^{k} W_{i}^{k}\right) P_{i}^{k \mid k}\left(F_{i}^{k}+M_{i}^{k} W_{i}^{k}\right)^{T}\right. \\
& \left.+2 \Gamma\left(P_{j}^{k \mid k}+\hat{x}_{j}^{k \mid k}\left(\hat{x}_{j}^{k \mid k}\right)^{T}\right) \Gamma^{T}\right),
\end{aligned}
$$

and

$$
\begin{aligned}
& \sum_{j=1}^{N} \sum_{p=1}^{N} \Delta \omega_{i j} \Delta \omega_{i p} \mathbb{E}\left\{\Theta_{i}^{k} \Gamma x_{j}^{k}\left(x_{p}^{k}\right)^{T} \Gamma^{T}\left(\Theta_{i}^{k}\right)^{T}\right\} \\
\leq & \frac{1}{2} \sum_{j=1}^{N} \sum_{p=1}^{N} \Delta \omega_{i j} \Delta \omega_{i p} \mathbb{E}\left\{\Theta_{i}^{k} \Gamma\left(x_{j}^{k}\left(x_{p}^{k}\right)^{T}+x_{p}^{k} x_{j, k}^{T}\right) \Gamma^{T}\left(\Theta_{i}^{k}\right)^{T}\right\} \\
\leq & \frac{1}{2} \sum_{j=1}^{N} \sum_{p=1}^{N} \Delta \omega_{i j} \Delta \omega_{i p} \mathbb{E}\left\{\Theta_{i}^{k} \Gamma\left(x_{j}^{k} x_{j, k}^{T}+x_{p}^{k}\left(x_{p}^{k}\right)^{T}\right) \Gamma^{T}\left(\Theta_{i}^{k}\right)^{T}\right\} \\
\leq & 2 \bar{\varsigma} \sum_{j=1}^{N} \varsigma_{j} \Gamma\left(P_{j}^{k \mid k}+\hat{x}_{j}^{k \mid k}\left(\hat{x}_{j}^{k \mid k}\right)^{T}\right) \Gamma^{T} .
\end{aligned}
$$

Similarly, we have

$$
\begin{aligned}
& \sum_{j=1}^{N} \sum_{p=1}^{N} \omega_{i j} \omega_{i p} \mathbb{E}\left\{\Theta_{i}^{k} \Gamma e_{p}^{k \mid k}\left(e_{j}^{k \mid k}\right)^{T} \Gamma^{T}\left(\Theta_{i}^{k}\right)^{T}\right\} \\
\leq & \bar{\omega}_{i} \sum_{j=1}^{N} \omega_{i j} \Gamma P_{j}^{k \mid k} \Gamma^{T},
\end{aligned}
$$

and

$$
\begin{aligned}
& \sum_{j=1}^{N} \sum_{p=1}^{N} \Delta \omega_{i j} \omega_{i p} \mathbb{E}\left\{\Theta_{i}^{k} \Gamma\left(x_{j}^{k}\left(e_{p}^{k \mid k}\right)^{T}+e_{p}^{k \mid k} x_{j, k}^{T}\right) \Gamma^{T}\left(\Theta_{i}^{k}\right)^{T}\right\} \\
\leq & \sum_{j=1}^{N} \sum_{p=1}^{N} \Delta \omega_{i j} \omega_{i p} \mathbb{E}\left\{\Theta _ { i } ^ { k } \Gamma \left(2 P_{j}^{k \mid k}+2 \hat{x}_{j}^{k \mid k}\left(\hat{x}_{j}^{k \mid k}\right)^{T}\right.\right. \\
& \left.\left.+P_{p}^{k \mid k}\right) \Gamma^{T}\left(\Theta_{i}^{k}\right)^{T}\right\} \\
\leq & 2 \bar{\omega}_{i} \sum_{j=1}^{N} \varsigma_{j} \Gamma\left(P_{j}^{k \mid k}+\hat{x}_{j}^{k \mid k}\left(\hat{x}_{j}^{k \mid k}\right)^{T}\right) \Gamma^{T}+\bar{\varsigma} \sum_{j=1}^{N} \omega_{i j} \Gamma P_{j}^{k \mid k} \Gamma^{T} .
\end{aligned}
$$

Substituting (33)-(37) into (17) yields

$$
\begin{aligned}
& P_{i}^{k+1 \mid k} \\
& \leq D_{i}^{k} R_{i \varpi}^{k}\left(D_{i}^{k}\right)^{T}+\left(1+\lambda_{1} \bar{\omega}_{i}+\bar{\varsigma}\right) \mathbb{E}\left\{\left(F_{i}^{k}+M_{i}^{k} W_{i}^{k}\right)\right. \\
& \left.P_{i}^{k \mid k}\left(F_{i}^{k}+M_{i}^{k} W_{i}^{k}\right)^{T}\right\}+\left(\lambda_{1}^{-1}+\bar{\varsigma}+\bar{\omega}_{i}\right) \sum_{j=1}^{N} \omega_{i j} \\
& \times \mathbb{E}\left\{\Gamma P_{j}^{k \mid k} \Gamma^{T}\right\}+2\left(1+\bar{\omega}_{i}+\bar{\varsigma}\right) \sum_{j=1}^{N} \varsigma_{j} \\
& \times \mathbb{E}\left\{\Gamma\left(P_{j}^{k \mid k}+\hat{x}_{j}^{k \mid k}\left(\hat{x}_{j}^{k \mid k}\right)^{T}\right) \Gamma^{T}\right\} \\
& \leq D_{i}^{k} R_{i \varpi}^{k}\left(D_{i}^{k}\right)^{T}+\left(1+\lambda_{1} \bar{\omega}_{i}+\bar{\varsigma}\right) \operatorname{tr}\left(F_{i}^{k}\left(\left(P_{i}^{k \mid k}\right)^{-1}-\eta I\right)^{-1}\right. \\
& \left.\times\left(F_{i}^{k}\right)^{T}+\eta^{-1} M_{i}^{k}\left(M_{i}^{k}\right)^{T}\right) I+\left(\lambda_{1}^{-1}+\bar{\varsigma}+\bar{\omega}_{i}\right) \sum_{j=1}^{N} \omega_{i j} \\
& \times \operatorname{tr}\left(\Gamma P_{j}^{k \mid k} \Gamma^{T}\right) I+2\left(1+\bar{\omega}_{i}+\bar{\varsigma}\right) \sum_{j=1}^{N} \varsigma_{j} \operatorname{tr}\left(\Gamma \left(P_{j}^{k \mid k}\right.\right.
\end{aligned}
$$

$$
\begin{aligned}
& \left.\left.+\hat{x}_{j}^{k \mid k}\left(\hat{x}_{j}^{k \mid k}\right)^{T}\right) \Gamma^{T}\right) I \\
= & s_{i}^{k} I+D_{i}^{k} R_{i \varpi}^{k}\left(D_{i}^{k}\right)^{T}
\end{aligned}
$$

where

$$
\begin{aligned}
s_{i}^{k} \triangleq & \left(1+\lambda_{1} \bar{\omega}_{i}+\bar{\varsigma}\right) \operatorname{tr}\left(F_{i}^{k}\left(\left(P_{i}^{k \mid k}\right)^{-1}-\eta I\right)^{-1}\left(F_{i}^{k}\right)^{T}+\eta^{-1}\right. \\
& \left.\times M_{i}^{k}\left(M_{i}^{k}\right)^{T}\right)+\left(\lambda_{1}^{-1}+\bar{\varsigma}+\bar{\omega}_{i}\right) \sum_{j=1}^{N} \omega_{i j} \operatorname{tr}\left(\Gamma P_{j}^{k \mid k} \Gamma^{T}\right) \\
& +2\left(1+\bar{\omega}_{i}+\bar{\varsigma}\right) \sum_{j=1}^{N} \varsigma_{j} \operatorname{tr}\left(\Gamma\left(P_{j}^{k \mid k}+\hat{x}_{j}^{k \mid k}\left(\hat{x}_{j}^{k \mid k}\right)^{T}\right) \Gamma^{T}\right) .
\end{aligned}
$$

Next, using the definition of $\sigma_{i}(\cdot)$ in (2), we have

$$
\begin{aligned}
& P_{i}^{k+1 \mid k} \\
= & D_{i}^{k} R_{i \varpi}^{k}\left(D_{i}^{k}\right)^{T}+\mathbb{E}\left\{\left(\sigma_{i}\left(f_{i}\left(x_{i}^{k}\right)+\sum_{j=1}^{N}\left(\omega_{i j}+\Delta \omega_{i j}\right) \Gamma x_{j}^{k}\right)\right.\right. \\
& \left.-\sigma_{i}\left(f_{i}\left(\hat{x}_{i}^{k \mid k}\right)+\sum_{j=1}^{N} \omega_{i j} \Gamma \hat{x}_{j}^{k \mid k}\right)\right)\left(\sigma _ { i } \left(f_{i}\left(x_{i}^{k}\right)+\sum_{j=1}^{N}\left(\omega_{i j}\right.\right.\right. \\
& \left.\left.\left.\left.+\Delta \omega_{i j}\right) \Gamma x_{j}^{k}\right)-\sigma_{i}\left(f_{i}\left(\hat{x}_{i}^{k \mid k}\right)+\sum_{j=1}^{N} \omega_{i j} \Gamma \hat{x}_{j}^{k \mid k}\right)\right)^{T}\right\} \\
\leq & 4 \bar{\pi}_{i} I+D_{i}^{k} R_{i \varpi}^{k}\left(D_{i}^{k}\right)^{T}
\end{aligned}
$$

where $\bar{\pi}_{i} \triangleq \sum_{h=1}^{n}\left(\pi_{i h}^{\max }\right)^{2}$.

From (38)-(40), it is concluded that

$$
P_{i}^{k+1 \mid k} \leq \min \left\{s_{i}^{k}, 4 \bar{\pi}_{i}\right\} I+D_{i}^{k} R_{i \varpi}^{k}\left(D_{i}^{k}\right)^{T} .
$$

Together with (26), (30), (41) and the assumption $P_{i}^{k \mid k} \leq$ $\Phi_{i}^{k \mid k}$, one assures that the inequality (28) holds.

By using Lemma 2 and the fact that $x_{i}^{k+1}=\hat{x}_{i}^{k+1 \mid k}+$ $e_{i}^{k+1 \mid k}$, one has

$$
\begin{aligned}
& P_{i}^{k+1 \mid k+1} \\
\leq & \left(I-K_{i}^{k+1} G_{i}^{k+1}\right) P_{i}^{k+1 \mid k}\left(I-K_{i}^{k+1} G_{i}^{k+1}\right)^{T} \\
& +\left(1-\bar{\alpha}_{i}\right) K_{i}^{k+1} R_{i v}^{k+1}\left(K_{i}^{k+1}\right)^{T}+\mathbb{E}\left\{\bar{\alpha}_{i} K_{i}^{k+1} \xi_{k+1} \xi_{k+1}^{T}\right. \\
& \times\left(K_{i}^{k+1}\right)^{T}+\bar{\alpha}_{i} K_{i}^{k+1} G_{i}^{k+1} x_{i}^{k+1}\left(K_{i}^{k+1} G_{i}^{k+1} x_{i}^{k+1}\right)^{T} \\
& +\bar{\alpha}_{i}\left(\rho_{1}\left(I-K_{i}^{k+1} G_{i}^{k+1}\right) P_{i}^{k+1 \mid k}\left(I-K_{i}^{k+1} G_{i}^{k+1}\right)^{T}\right. \\
& \left.+\rho_{1}^{-1} K_{i}^{k+1} G_{i}^{k+1} x_{i}^{k+1}\left(K_{i}^{k+1} G_{i}^{k+1} x_{i}^{k+1}\right)^{T}\right) \\
& +\bar{\alpha}_{i}\left(\rho_{2} K_{i}^{k+1} G_{i}^{k+1} x_{i}^{k+1}\left(K_{i}^{k+1} G_{i}^{k+1} x_{i}^{k+1}\right)^{T}\right. \\
& \left.+\rho_{2}^{-1} K_{i}^{k+1} \xi_{k+1} \xi_{k+1}^{T}\left(K_{i}^{k+1}\right)^{T}\right)+\bar{\alpha}_{i}\left(\rho _ { 3 } \left(I-K_{i}^{k+1}\right.\right. \\
& \left.\times G_{i}^{k+1}\right) P_{i}^{k+1 \mid k}\left(I-K_{i}^{k+1} G_{i}^{k+1}\right)^{T} \\
& \left.\left.+\rho_{3}^{-1} K_{i}^{k+1} \xi_{k+1} \xi_{k+1}^{T}\left(K_{i}^{k+1}\right)^{T}\right)\right\} \\
\leq & \left(1+\bar{\alpha}_{i} \rho_{1}+\bar{\alpha}_{i} \rho_{3}\right)\left(I-K_{i}^{k+1} G_{i}^{k+1}\right) P_{i}^{k+1 \mid k} \\
& \times\left(I-K_{i}^{k+1} G_{i}^{k+1}\right)^{T}+\left(1-\bar{\alpha}_{i}\right) K_{i}^{k+1} R_{i v}^{k+1}\left(K_{i}^{k+1}\right)^{T} \\
& +\bar{\alpha}_{i}\left(1+\rho_{2}^{-1}+\rho_{3}^{-1}\right) \theta^{2} K_{i}^{k+1}\left(K_{i}^{k+1}\right)^{T} \\
& +2 \bar{\alpha}_{i}\left(1+\rho_{1}^{-1}+\rho_{2}\right) K_{i}^{k+1} G_{i}^{k+1} P_{i}^{k+1 \mid k}\left(G_{i}^{k+1}\right)^{T}\left(K_{i}^{k+1}\right)^{T} \\
& +2 \bar{\alpha}_{i}\left(1+\rho_{1}^{-1}+\rho_{2}\right) K_{i}^{k+1} G_{i}^{k+1} \hat{x}_{i}^{k+1 \mid k}\left(\hat{x}_{i}^{k+1 \mid k}\right)^{T} \\
& \times\left(G_{i}^{k+1}\right)^{T}\left(K_{i}^{k+1}\right)^{T} .
\end{aligned}
$$


This article has been accepted for publication in a future issue of this journal, but has not been fully edited. Content may change prior to final publication. Citation information: DOI 10.1109/TCYB.2021.3067822, IEEE Transactions on Cybernetics

Applying Lemma 4 to (27) and (42), we draw a conclusion as (29).

From the above discussions, the upper bounds (27) on $P_{i}^{k+1 \mid k+1}$ are obtained. Moving forward, we arrive at

$$
\begin{aligned}
& \frac{\partial \operatorname{tr}\left(\Phi_{i}^{k+1 \mid k+1}\right)}{\partial K_{i}^{k+1}} \\
= & 2\left(1+\bar{\alpha}_{i} \rho_{1}+\bar{\alpha}_{i} \rho_{3}\right)\left(-\Phi_{i}^{k+1 \mid k}\left(G_{i}^{k+1}\right)^{T}+K_{i}^{k+1} G_{i}^{k+1}\right. \\
& \left.\times \Phi_{i}^{k+1 \mid k}\left(G_{i}^{k+1}\right)^{T}\right)+4 \bar{\alpha}_{i}\left(1+\rho_{1}^{-1}+\rho_{2}\right) K_{i}^{k+1} G_{i}^{k+1} \\
& \times \Phi_{i}^{k+1 \mid k}\left(G_{i}^{k+1}\right)^{T}+2 \bar{\alpha}_{i}\left(1+\rho_{2}^{-1}+\rho_{3}^{-1}\right) \theta^{2} K_{i}^{k+1} \\
& +4 \bar{\alpha}_{i}\left(1+\rho_{1}^{-1}+\rho_{2}\right) K_{i}^{k+1} G_{i}^{k+1} \hat{x}_{i}^{k+1 \mid k}\left(\hat{x}_{i}^{k+1 \mid k}\right)^{T}\left(G_{i}^{k+1}\right)^{T} \\
& +2\left(1-\bar{\alpha}_{i}\right) K_{i}^{k+1} R_{i v}^{k+1} \\
= & -2\left(1+\bar{\alpha}_{i} \rho_{1}+\bar{\alpha}_{i} \rho_{3}\right) \Phi_{i}^{k+1 \mid k}\left(G_{i}^{k+1}\right)^{T}+2 K_{i}^{k+1} \Pi_{i}^{k+1}
\end{aligned}
$$$$
=0 \text {. }
$$

It is obtained from (43) and the invertibility of $\Pi_{i}^{k+1}$ that $K_{i}^{k+1}$ is computed by (31).

Remark 2: In this paper, a novel approach has been presented to calculate the time-varying filter gain for CNs. The main idea of this method is to calculate the filter gain of node $i$ by only using its local and neighboring information, thereby reducing the computational complexity. Note that, by using the traditional state-augmentation method, the dimension of the corresponding upper-bound matrix amounts to $n N \times n N$, which would give rise to a heavy computational burden in case of large network size. As such, our recursive scheme is more suitable for online application in practical engineering as compared with the traditional state augmentation approach.

Now, we are ready to discuss the EMSB of $e_{i}^{k \mid k}$ under Assumption 1 and Lemmas 7-8.

Assumption 1: The positive scalars $\underline{r}_{i \varpi}, \bar{r}_{i \varpi}, \underline{\gamma}, \bar{\gamma}, \underline{d}_{i}, \bar{d}_{i}$, $\underline{f}_{i}, \bar{f}_{i}, \underline{m}_{i}$ and $\bar{m}_{i}$ satisfy

$$
\begin{gathered}
\underline{r}_{i \varpi} \leq\left\|R_{i \varpi, k+1}\right\| \leq \bar{r}_{i \varpi}, \quad \underline{\gamma} \leq\|\Gamma\| \leq \bar{\gamma}, \\
\underline{d}_{i} \leq\left\|D_{i}^{k}\right\| \leq \bar{d}_{i}, \underline{f}_{i} \leq\left\|F_{i}^{k}\right\| \leq \overline{\bar{f}}_{i}, \underline{m}_{i} \leq\left\|M_{i}^{k}\right\| \leq \bar{m}_{i}
\end{gathered}
$$

for any $i$ and $k$.

Lemma 7: Consider the state-saturated $\mathrm{CN}$ described by (1)-(6) with filter (7). Then, we have

$$
\begin{aligned}
\mathcal{H}_{i}^{k+1} & \leq\left(\Phi_{i}^{k+1 \mid k}\right)^{-1} \\
\left(G_{i}^{k+1}\right)^{T} \mathcal{K}_{i}^{k+1} G_{i}^{k+1} & \leq \frac{1}{2 \bar{\alpha}_{i}\left(1+\rho_{1}^{-1}+\rho_{2}\right)}\left(\Phi_{i}^{k+1 \mid k}\right)^{-1} \\
\left(\Phi_{i}^{k+1 \mid k}\right)^{-1} & \leq \frac{1}{\bar{d}_{i}^{2} \bar{r}_{i \varpi}} I
\end{aligned}
$$

if positive scalars $\rho_{1}$ and $\rho_{2}$ exist such that

$$
\begin{aligned}
3+2 \rho_{1}^{-1}+2 \rho_{2} & \geq \rho_{1}, \\
\rho_{1} & \geq 2
\end{aligned}
$$

where

$$
\begin{aligned}
& \mathcal{H}_{i}^{k+1} \triangleq\left(\mathcal{L}_{i}^{k+1}\right)^{T}\left(\Phi_{i}^{k+1 \mid k+1}\right)^{-1} \mathcal{L}_{i}^{k+1}, \\
& \mathcal{K}_{i}^{k+1} \triangleq\left(K_{i}^{k+1}\right)^{T} \times\left(\Phi_{i}^{k+1 \mid k+1}\right)^{-1} K_{i}^{k+1}, \\
& \mathcal{L}_{i}^{k+1} \triangleq I-\left(1-\bar{\alpha}_{i}\right) K_{i}^{k+1} G_{i}^{k+1} .
\end{aligned}
$$

Proof: It follows from (26) that

$$
\Phi_{i}^{k+1 \mid k} \geq D_{i}^{k} R_{i \varpi}^{k}\left(D_{i}^{k}\right)^{T},
$$

or

$$
\left(\Phi_{i}^{k+1 \mid k}\right)^{-1} \leq \frac{1}{\bar{d}_{i}^{2} \bar{r}_{i \varpi}} I .
$$

From (27), one knows that

$$
\begin{aligned}
\Phi_{i}^{k+1 \mid k+1} \geq & \left(1+\bar{\alpha}_{i} \rho_{1}+\bar{\alpha}_{i} \rho_{3}\right)\left(I-K_{i}^{k+1} G_{i}^{k+1}\right) \Phi_{i}^{k+1 \mid k} \\
& \times\left(I-K_{i}^{k+1} G_{i}^{k+1}\right)^{T}+2 \bar{\alpha}_{i}\left(1+\rho_{1}^{-1}+\rho_{2}\right) \\
& \times K_{i}^{k+1} G_{i}^{k+1} \Phi_{i}^{k+1 \mid k}\left(G_{i}^{k+1}\right)^{T}\left(K_{i}^{k+1}\right)^{T} .
\end{aligned}
$$

Thus, if the positive scalars $\rho_{1}$ and $\rho_{2}$ satisfy the condition (45), the inequality

$$
\Phi_{i}^{k+1 \mid k+1} \geq \mathcal{L}_{i}^{k+1} \Phi_{i}^{k+1 \mid k}\left(\mathcal{L}_{i}^{k+1}\right)^{T},
$$

holds. Then, by applying Lemma 5, the above inequality (48) holds if and only if

$$
\left(\mathcal{L}_{i}^{k+1}\right)^{T}\left(\Phi_{i}^{k+1 \mid k+1}\right)^{-1} \mathcal{L}_{i}^{k+1} \leq\left(\Phi_{i}^{k+1 \mid k}\right)^{-1},
$$

i.e., $\mathcal{H}_{i}^{k+1} \leq\left(\Phi_{i}^{k+1 \mid k}\right)^{-1}$.

In addition, (27) tells that

$$
\begin{aligned}
\Phi_{i}^{k+1 \mid k+1} \geq & 2 \bar{\alpha}_{i}\left(1+\rho_{1}^{-1}+\rho_{2}\right) K_{i}^{k+1} G_{i}^{k+1} \Phi_{i}^{k+1 \mid k} \\
& \times\left(G_{i}^{k+1}\right)^{T}\left(K_{i}^{k+1}\right)^{T} .
\end{aligned}
$$

By means of Lemma 5, it is derived from (50) that

$$
\begin{aligned}
& \left(G_{i}^{k+1}\right)^{T}\left(K_{i}^{k+1}\right)^{T}\left(\Phi_{i}^{k+1 \mid k+1}\right)^{-1} K_{i}^{k+1} G_{i}^{k+1} \\
\leq & \frac{1}{2 \bar{\alpha}_{i}\left(1+\rho_{1}^{-1}+\rho_{2}\right)}\left(\Phi_{i}^{k+1 \mid k}\right)^{-1},
\end{aligned}
$$

or

$$
\left(G_{i}^{k+1}\right)^{T} \mathcal{K}_{i}^{k+1} G_{i}^{k+1} \leq \frac{1}{2 \bar{\alpha}_{i}\left(1+\rho_{1}^{-1}+\rho_{2}\right)}\left(\Phi_{i}^{k+1 \mid k}\right)^{-1} .
$$

Based on Assumption 1 and summarizing the above analysis, Lemma 7 is proven.

Lemma 8: Consider the state-saturated $\mathrm{CN}$ described by (1)-(6). We have

$$
\begin{aligned}
& \mathcal{F}_{i, k}^{T}\left(\Phi_{i}^{k+1 \mid k}\right)^{-1} \mathcal{F}_{i, k} \leq \frac{1}{1+\lambda_{1} \bar{\omega}_{i}+\bar{\varsigma}}\left(\Phi_{i}^{k \mid k}\right)^{-1} \\
& \left(\Phi_{i}^{k+1 \mid k}\right)^{-1} \leq \frac{1}{1+\lambda_{1} \bar{\omega}_{i}+\bar{\varsigma}}\left(\left(F_{i}^{k}\right)^{T}\right)^{-1}\left(\Phi_{i}^{k \mid k}\right)^{-1}\left(F_{i}^{k}\right)^{-1}
\end{aligned}
$$

when $\Phi_{i}^{k+1 \mid k}=z_{i}^{k} I+D_{i}^{k} R_{i \varpi}^{k}\left(D_{i}^{k}\right)^{T}$, where $\mathcal{F}_{i, k} \triangleq F_{i}^{k}+$ $M_{i}^{k} W_{i}^{k}$.

Proof: From (26), (30) and Lemma 3, we can easily obtain the following inequalities:

$$
\begin{aligned}
& \Phi_{i}^{k+1 \mid k} \geq\left(1+\lambda_{1} \bar{\omega}_{i}+\bar{\varsigma}\right) \mathcal{F}_{i, k} \Phi_{i}^{k \mid k} \mathcal{F}_{i, k}^{T}, \\
& \Phi_{i}^{k+1 \mid k} \geq\left(1+\lambda_{1} \bar{\omega}_{i}+\bar{\varsigma}\right) F_{i}^{k}\left(\left(\Phi_{i}^{k \mid k}\right)^{-1}-\eta I\right)^{-1}\left(F_{i}^{k}\right)^{T} .
\end{aligned}
$$

According to the definition of $F_{i}^{k}$ in (20), it is easy to know that $F_{i}^{k}$ is invertible. Based on Lemma 5, we see from (53) that Lemma 8 holds. 
This article has been accepted for publication in a future issue of this journal, but has not been fully edited. Content may change prior to final publication. Citation information: DOI 10.1109/TCYB.2021.3067822, IEEE Transactions on Cybernetics

Theorem 3: Consider the state-saturated $\mathrm{CN}$ depicted by (1). Let $\lambda_{1}, \rho_{1}$ and $\rho_{2}$ be positive scalars satisfying

$$
\begin{aligned}
2 \rho_{2} & \geq \rho_{1}-2 \rho_{1}^{-1}-3, \\
\rho_{1} & \geq 2 \\
\lambda_{1} \bar{\omega}_{i} \underline{f}_{i}^{2}>\underline{f}_{i}^{2}\left(5+3 \bar{\varsigma}+2 \bar{\omega}_{i}\right)+N \bar{f}_{i}^{2} \bar{\gamma}^{2}\left(\bar{\varsigma}+\bar{\omega}_{i}\right) & \quad \times\left(5+3 N \bar{\varsigma}+2 N \bar{\omega}_{i}\right) .
\end{aligned}
$$

Then, the EMSB of $e_{i}^{k \mid k}$ is ensured under Assumption 1.

Proof: First, we define the following notations:

$$
e^{k+1 \mid k} \triangleq\left[\left(e_{1}^{k+1 \mid k}\right)^{T},\left(e_{2}^{k+1 \mid k}\right)^{T}, \cdots,\left(e_{N}^{k+1 \mid k}\right)^{T}\right]^{T},
$$

$e^{k+1 \mid k+1} \triangleq\left[\left(e_{1}^{k+1 \mid k+1}\right)^{T},\left(e_{2}^{k+1 \mid k+1}\right)^{T}, \cdots,\left(e_{N}^{k+1 \mid k+1}\right)^{T}\right]^{T}$.

Next, we select a quadratic function as follows:

$$
V_{k}\left(e^{k \mid k}\right) \triangleq \sum_{i=1}^{N}\left(e_{i}^{k \mid k}\right)^{T}\left(\Phi_{i}^{k \mid k}\right)^{-1} e_{i}^{k \mid k} .
$$

Substituting (22) and (24) into (55), we obtain

$$
\begin{aligned}
& \mathbb{E}\left\{V_{k+1}\left(e^{k+1 \mid k+1}\right) \mid e^{k \mid k}\right\} \\
= & \sum_{i=1}^{N}\left(e_{i}^{k+1 \mid k+1}\right)^{T}\left(\Phi_{i}^{k+1 \mid k+1}\right)^{-1} e_{i}^{k+1 \mid k+1} .
\end{aligned}
$$

According to (22), (24) and recalling $x_{i}^{k+1}=e_{i}^{k+1 \mid k}+$ $\hat{x}_{i}^{k+1 \mid k}$, one has

$$
\begin{aligned}
e_{i}^{k+1 \mid k+1}= & \left(\mathcal{L}_{i}^{k+1}+\tilde{\alpha}_{i}^{k+1} K_{i}^{k+1} G_{i}^{k+1}\right) \Theta_{i}^{k}\left(\mathcal{F}_{i, k} e_{i}^{k \mid k}\right. \\
& \left.+\sum_{j=1}^{N} \Delta \omega_{i j} \Gamma \hat{x}_{j}^{k \mid k}+\sum_{j=1}^{N}\left(\Delta \omega_{i j}+\omega_{i j}\right) \Gamma e_{j}^{k \mid k}\right) \\
& +\left(\mathcal{L}_{i}^{k+1}+\tilde{\alpha}_{i}^{k+1} K_{i}^{k+1} G_{i}^{k+1}\right) D_{i}^{k} \varpi_{i}^{k} \\
& +\bar{\alpha}_{i} K_{i}^{k+1} G_{i}^{k+1} \hat{x}_{i}^{k+1 \mid k}-\left(1-\bar{\alpha}_{i}\right) K_{i}^{k+1} v_{i}^{k+1} \\
& -\bar{\alpha}_{i} K_{i}^{k+1} \xi_{k+1}+\tilde{\alpha}_{i}^{k+1} K_{i}^{k+1}\left(G_{i}^{k+1} \hat{x}_{i}^{k+1 \mid k}\right. \\
& \left.+v_{i}^{k+1}-\xi_{k+1}\right) .
\end{aligned}
$$

Calculate $\mathbb{E}\left\{V_{k+1}\left(e^{k+1 \mid k+1}\right) \mid e^{k \mid k}\right\}$ along (57) as follows:

$$
\begin{aligned}
& \mathbb{E}\left\{V_{k+1}\left(e^{k+1 \mid k+1}\right) \mid e^{k \mid k}\right\} \\
= & \sum_{i=1}^{N}\left(e_{i}^{k \mid k}\right)^{T} \mathcal{F}_{i, k}^{T}\left(\Theta_{i}^{k}\right)^{T} \mathcal{G}_{i}^{k+1} \Theta_{i}^{k} \mathcal{F}_{i, k} e_{i}^{k \mid k}+\sum_{i=1}^{N} \sum_{j=1}^{N} \sum_{h=1}^{n}\left(\Delta \omega_{i j}\right. \\
& \left.+\omega_{i j}\right)\left(\Delta \omega_{i h}+\omega_{i h}\right)\left(e_{j}^{k \mid k}\right)^{T} \Gamma^{T}\left(\Theta_{i}^{k}\right)^{T} \mathcal{G}_{i}^{k+1} \Theta_{i}^{k} \Gamma e_{h}^{k \mid k} \\
& +\sum_{i=1}^{N} \sum_{j=1}^{N} \sum_{h=1}^{n} \Delta \omega_{i j} \Delta \omega_{i h}\left(\hat{x}_{j}^{k \mid k}\right)^{T} \Gamma^{T}\left(\Theta_{i}^{k}\right)^{T} \mathcal{G}_{i}^{k+1} \Theta_{i}^{k} \Gamma \hat{x}_{h}^{k \mid k} \\
& +\sum_{i=1}^{N} \bar{\alpha}_{i} \xi_{k+1}^{T} \mathcal{K}_{i}^{k+1} \xi_{k+1}+\sum_{i=1}^{N}\left(\varpi_{i}^{k}\right)^{T}\left(D_{i}^{k}\right)^{T} \mathcal{G}_{i}^{k+1} D_{i}^{k} \varpi_{i}^{k} \\
& +\sum_{i=1}^{N} \bar{\alpha}_{i}\left(\hat{x}_{i}^{k+1 \mid k}\right)^{T}\left(G_{i}^{k+1}\right)^{T} \mathcal{K}_{i}^{k+1} G_{i}^{k+1} \hat{x}_{i}^{k+1 \mid k} \\
& +\sum_{i=1}^{N}\left(1-\bar{\alpha}_{i}\right)\left(v_{i}^{k+1}\right)^{T} \mathcal{K}_{i}^{k+1} v_{i}^{k+1} \\
& +2 \sum_{i=1}^{N} \sum_{j=1}^{N}\left(\Delta \omega_{i j}+\omega_{i j}\right)\left(e_{i}^{k \mid k}\right)^{T} \mathcal{F}_{i, k}^{T}\left(\Theta_{i}^{k}\right)^{T} \mathcal{G}_{i}^{k+1} \Theta_{i}^{k} \Gamma e_{j}^{k \mid k}
\end{aligned}
$$

$$
\begin{aligned}
& +2 \sum_{i=1}^{N} \sum_{j=1}^{N} \Delta \omega_{i j}\left(e_{i}^{k \mid k}\right)^{T} \mathcal{F}_{i, k}^{T}\left(\Theta_{i}^{k}\right)^{T} \mathcal{G}_{i}^{k+1} \Theta_{i}^{k} \Gamma \hat{x}_{j}^{k \mid k} \\
& +2 \sum_{i=1}^{N} \bar{\alpha}_{i}\left(e_{i}^{k \mid k}\right)^{T} \mathcal{F}_{i, k}^{T}\left(\Theta_{i}^{k}\right)^{T} \mathcal{Q}_{i}^{k+1}\left(G_{i}^{k+1} \hat{x}_{i}^{k+1 \mid k}-\xi_{k+1}\right) \\
& -2 \sum_{i=1}^{N} \bar{\alpha}_{i}\left(\hat{x}_{i}^{k+1 \mid k}\right)^{T}\left(G_{i}^{k+1}\right)^{T} \mathcal{K}_{i}^{k+1} \xi_{k+1} \\
& +2 \sum_{i=1}^{N} \sum_{j=1}^{N} \sum_{h=1}^{N} \Delta \omega_{i h}\left(\Delta \omega_{i j}+\omega_{i j}\right)\left(e_{j}^{k \mid k}\right)^{T} \Gamma^{T}\left(\Theta_{i}^{k}\right)^{T} \mathcal{G}_{i}^{k+1} \\
& \times \Theta_{i}^{k} \Gamma \hat{x}_{h}^{k \mid k} \hat{x}_{h}^{k \mid k}+2 \sum_{i=1}^{N} \sum_{j=1}^{N} \bar{\alpha}_{i}\left(\Delta \omega_{i j}+\omega_{i j}\right)\left(e_{j}^{k \mid k}\right)^{T} \Gamma^{T} \\
& \times\left(\Theta_{i}^{k}\right)^{T} \mathcal{Q}_{i}^{k+1}\left(G_{i}^{k+1} \hat{x}_{i}^{k+1 \mid k}-\xi_{k+1}\right)+2 \sum_{i=1}^{N} \sum_{j=1}^{N} \bar{\alpha}_{i} \Delta \omega_{i j} \\
& \times\left(\hat{x}_{j}^{k \mid k}\right)^{T} \Gamma^{T}\left(\Theta_{i}^{k}\right)^{T} \mathcal{Q}_{i}^{k+1}\left(G_{i}^{k+1} \hat{x}_{i}^{k+1 \mid k}-\xi_{k+1}\right)+2 \sum_{i=1}^{N} \bar{\alpha}_{i} \\
& \times\left(1-\bar{\alpha}_{i}\right)\left(e_{i}^{k \mid k}\right)^{T} \mathcal{F}_{i, k}^{T}\left(\Theta_{i}^{k}\right)^{T}\left(G_{i}^{k+1}\right)^{T} \mathcal{K}_{i}^{k+1}\left(G_{i}^{k+1} \hat{x}_{i}^{k+1 \mid k}\right. \\
& \left.-\xi_{k+1}\right)+2 \sum_{i=1}^{N} \sum_{j=1}^{N} \bar{\alpha}_{i}\left(1-\bar{\alpha}_{i}\right) \Delta \omega_{i j}\left(\hat{x}_{j}^{k \mid k}\right)^{T} \Gamma^{T}\left(\Theta_{i}^{k}\right)^{T} \\
& \times\left(G_{i}^{k+1}\right)^{T} \mathcal{K}_{i}^{k+1}\left(G_{i}^{k+1} \hat{x}_{i}^{k+1 \mid k}-\xi_{k+1}\right) \\
& +2 \sum_{i=1}^{N} \sum_{j=1}^{N} \bar{\alpha}_{i}\left(1-\bar{\alpha}_{i}\right)\left(\Delta \omega_{i j}+\omega_{i j}\right)\left(e_{j}^{k \mid k}\right)^{T} \Gamma^{T}\left(\Theta_{i}^{k}\right)^{T} \\
& \times\left(G_{i}^{k+1}\right)^{T} \mathcal{K}_{i}^{k+1}\left(G_{i}^{k+1} \hat{x}_{i}^{k+1 \mid k}-\xi_{k+1}\right)
\end{aligned}
$$

where $\mathcal{Q}_{i}^{k+1} \triangleq\left(\mathcal{L}_{i}^{k+1}\right)^{T}\left(\Phi_{i}^{k+1 \mid k+1}\right)^{-1} K_{i}^{k+1}$ and $\mathcal{G}_{i}^{k+1} \triangleq$ $\mathcal{H}_{i}^{k+1}+\bar{\alpha}_{i}\left(1-\bar{\alpha}_{i}\right)\left(G_{i}^{k+1}\right)^{T} \mathcal{K}_{i}^{k+1} G_{i}^{k+1}$.

Observing the terms in (58), one has

$$
\begin{aligned}
& \sum_{i=1}^{N} \sum_{j=1}^{N} \sum_{h=1}^{n}\left(\Delta \omega_{i j}+\omega_{i j}\right)\left(\Delta \omega_{i h}+\omega_{i h}\right)\left(e_{j}^{k \mid k}\right)^{T} \Gamma^{T} \\
& \times\left(\Theta_{i}^{k}\right)^{T} \mathcal{G}_{i}^{k+1} \Theta_{i}^{k} \Gamma e_{h}^{k \mid k} \\
\leq & \sum_{i=1}^{N} \sum_{j=1}^{N} N\left(\Delta \omega_{i j}+\omega_{i j}\right)^{2}\left(e_{j}^{k \mid k}\right)^{T} \Gamma^{T}\left(\Theta_{i}^{k}\right)^{T} \mathcal{G}_{i}^{k+1} \Theta_{i}^{k} \Gamma e_{j}^{k \mid k} \\
\leq & \sum_{i=1}^{N} \sum_{j=1}^{N} N\left(\varsigma_{j}+\omega_{i j}\right)^{2}\left(e_{j}^{k \mid k}\right)^{T} \Gamma^{T} \mathcal{G}_{i}^{k+1} \Gamma e_{j}^{k \mid k}
\end{aligned}
$$

and

$$
\begin{aligned}
& \sum_{i=1}^{N} \sum_{j=1}^{N} \sum_{h=1}^{n} \Delta \omega_{i j} \Delta \omega_{i h}\left(\hat{x}_{j}^{k \mid k}\right)^{T} \Gamma^{T}\left(\Theta_{i}^{k}\right)^{T} \mathcal{G}_{i}^{k+1} \Theta_{i}^{k} \Gamma \hat{x}_{h}^{k \mid k} \\
\leq & \sum_{i=1}^{N} \sum_{j=1}^{N} N \varsigma_{j}^{2}\left(\hat{x}_{j}^{k \mid k}\right)^{T} \Gamma^{T} \mathcal{G}_{i}^{k+1} \Gamma \hat{x}_{j}^{k \mid k}
\end{aligned}
$$

For the cross terms in (58), one has

$2 \sum_{i=1}^{N} \sum_{j=1}^{N}\left(\Delta \omega_{i j}+\omega_{i j}\right)\left(e_{i}^{k \mid k}\right)^{T} \mathcal{F}_{i, k}^{T}\left(\Theta_{i}^{k}\right)^{T}\left(\mathcal{G}_{i}^{k+1}\right)^{T} \Theta_{i}^{k} \Gamma e_{j}^{k \mid k}$ 
This article has been accepted for publication in a future issue of this journal, but has not been fully edited. Content may change prior to final publication. Citation information: DOI 10.1109/TCYB.2021.3067822, IEEE Transactions on Cybernetics

$$
\begin{aligned}
\leq & \sum_{i=1}^{N} \sum_{j=1}^{N}\left(\varsigma_{j}+\omega_{i j}\right)\left(e_{i}^{k \mid k}\right)^{T} \mathcal{F}_{i, k}^{T} \mathcal{G}_{i}^{k+1} \mathcal{F}_{i, k} e_{i}^{k \mid k} \\
& +\sum_{i=1}^{N} \sum_{j=1}^{N}\left(\varsigma_{j}+\omega_{i j}\right)\left(e_{j}^{k \mid k}\right)^{T} \Gamma^{T} \mathcal{G}_{i}^{k+1} \Gamma e_{j}^{k \mid k} .
\end{aligned}
$$

The remaining cross terms in (58) can be handled similarly. Moreover, substituting (59)-(61) and all the other cross terms into (58), we have

$$
\begin{aligned}
& \mathbb{E}\left\{V_{k+1}\left(e^{k+1 \mid k+1}\right) \mid e^{k \mid k}\right\} \\
\leq & \sum_{i=1}^{N}\left(e_{i}^{k \mid k}\right)^{T} \mathcal{F}_{i, k}^{T}\left(\left(1+2 \bar{\varsigma}+\bar{\omega}_{i}+2 \bar{\alpha}_{i}\right) \mathcal{H}_{i}^{k+1}+\bar{\alpha}_{i}\left(1-\bar{\alpha}_{i}\right)\right. \\
& \left.\times\left(3+2 \bar{\varsigma}+\bar{\omega}_{i}\right)\left(G_{i}^{k+1}\right)^{T} \mathcal{K}_{i}^{k+1} G_{i}^{k+1}\right) \mathcal{F}_{i, k} e_{i}^{k \mid k} \\
& +\sum_{i=1}^{N} \sum_{j=1}^{N}\left(\varsigma_{j}+\omega_{i j}\right)\left(e_{j}^{k \mid k}\right)^{T} \Gamma^{T}\left(\left(1+2 N \varsigma_{j}+N \omega_{i j}+2 \bar{\alpha}_{i}\right)\right. \\
& \times \mathcal{H}_{i}^{k+1}+\bar{\alpha}_{i}\left(1-\bar{\alpha}_{i}\right)\left(3+2 N \varsigma_{j}+N \omega_{i j}\right)\left(G_{i}^{k+1}\right)^{T} \mathcal{K}_{i}^{k+1} \\
& \left.\times G_{i}^{k+1}\right) \Gamma e_{j}^{k \mid k}+\sum_{i=1}^{N} \bar{\alpha}_{i}\left(2+\left(1+2 \bar{\varsigma}+\bar{\omega}_{i}\right)\left(2-\bar{\alpha}_{i}\right)\right) \\
& \times\left(\xi_{k+1}^{T} \mathcal{K}_{i}^{k+1} \xi_{k+1}+\left(\hat{x}_{i}^{k+1 \mid k}\right)^{T}\left(G_{i}^{k+1}\right)^{T} \mathcal{K}_{i}^{k+1} G_{i}^{k+1} \hat{x}_{i}^{k+1 \mid k}\right) \\
& +\sum_{i=1}^{N} \sum_{j=1}^{N} \varsigma_{j}\left(\hat{x}_{j}^{k \mid k}\right)^{T} \Gamma^{T}\left(\left(1+2 N \varsigma_{j}+N \omega_{i j}+2 \bar{\alpha}_{i}\right) \mathcal{H}_{i}^{k+1}\right. \\
& \left.+\bar{\alpha}_{i}\left(1-\bar{\alpha}_{i}\right)\left(3+2 N \varsigma_{j}+N \omega_{i j}\right)\left(G_{i}^{k+1}\right)^{T} \mathcal{K}_{i}^{k+1} G_{i}^{k+1}\right) \\
& \times \Gamma \hat{x}_{j}^{k \mid k}+\sum_{i=1}^{N}\left(\varpi_{i}^{k}\right)^{T}\left(D_{i}^{k}\right)^{T} \mathcal{G}_{i}^{k+1} D_{i}^{k} \varpi_{i}^{k} \\
& +\sum_{i=1}^{N}\left(1-\bar{\alpha}_{i}\right)\left(v_{i}^{k+1}\right)^{T} \mathcal{K}_{i}^{k+1} v_{i}^{k+1} . \\
& N{ }^{k}{ }_{i}
\end{aligned}
$$

Now, let us focus on the terms of $\mathcal{H}_{i}^{k+1}$ and $\mathcal{K}_{i}^{k+1}$ in (62) by recalling the inequalities (44) in Lemma 7, in which the matrix $\Phi_{i}^{k+1 \mid k}$ has two possible solutions according to Theorem 2. As such, we shall discuss the EMSB of $e_{i}^{k \mid k}$ for both cases.

Case 1: Consider the case that $\Phi_{i}^{k+1 \mid k}=4 \bar{\pi}_{i} I+$ $D_{i}^{k} R_{i \varpi}^{k}\left(D_{i}^{k}\right)^{T}$. It is obvious from Lemma 7 that $\left(\Phi_{i}^{k+1 \mid k}\right)^{-1}$, $\mathcal{H}_{i}^{k+1}$ and $\mathcal{K}_{i}^{k+1}$ are all bounded. According to (1) and (7), we know that $\hat{x}_{i}^{k+1 \mid k}$ and $\hat{x}_{i}^{k \mid k}$ are also both bounded. To sum up, the right-hand side of inequality (62) is bounded based on Assumption 1, and thus $e_{i}^{k \mid k}$ has the EMSB from Lemma 6.

Case 2: Consider the case that $\Phi_{i}^{k+1 \mid k}=z_{i}^{k} I+$ $D_{i}^{k} R_{i \varpi}^{k}\left(D_{i}^{k}\right)^{T}$. Let us recall Lemmas 7-8 and Assumption 1 , and then substitute (44) into (62). The inequality (62) is arranged as

$$
\begin{aligned}
& \mathbb{E}\left\{V_{k+1}\left(e^{k+1 \mid k+1}\right) \mid e^{k \mid k}\right\} \\
\leq & \sum_{i=1}^{N}\left(e_{i}^{k \mid k}\right)^{T}\left(\frac{1+2 \bar{\varsigma}+\bar{\omega}_{i}+2 \bar{\alpha}_{i}}{1+\lambda_{1} \bar{\omega}_{i}+\bar{\varsigma}}\left(\Phi_{i}^{k \mid k}\right)^{-1}\right. \\
& \left.+\frac{\left(1-\bar{\alpha}_{i}\right)\left(3+2 \bar{\varsigma}+\bar{\omega}_{i}\right)}{2\left(1+\rho_{1}^{-1}+\rho_{2}\right)\left(1+\lambda_{1} \bar{\omega}_{i}+\bar{\varsigma}\right)}\left(\Phi_{i}^{k \mid k}\right)^{-1}\right) e_{i}^{k \mid k} \\
& +N \sum_{i=1}^{N}\left(\bar{\varsigma}+\bar{\omega}_{i}\right)\left(e_{i}^{k \mid k}\right)^{T} \Gamma^{T}\left(\frac{1+2 N \bar{\varsigma}+N \bar{\omega}_{i}+2 \bar{\alpha}_{i}}{1+\lambda_{1} \bar{\omega}_{i}+\bar{\varsigma}}\right.
\end{aligned}
$$

$$
\begin{aligned}
& \times\left(\left(F_{i}^{k}\right)^{T}\right)^{-1}\left(\Phi_{i}^{k \mid k}\right)^{-1}\left(F_{i}^{k}\right)^{-1} \\
& +\frac{\left(1-\bar{\alpha}_{i}\right)\left(3+2 N \bar{\varsigma}+N \bar{\omega}_{i}\right)}{2\left(1+\rho_{1}^{-1}+\rho_{2}\right)\left(1+\lambda_{1} \bar{\omega}_{i}+\bar{\varsigma}\right)} \\
& \left.\times\left(\left(F_{i}^{k}\right)^{T}\right)^{-1}\left(\Phi_{i}^{k \mid k}\right)^{-1}\left(F_{i}^{k}\right)^{-1}\right) \Gamma e_{i}^{k \mid k} \\
& +\sum_{i=1}^{N} \frac{2+\left(1+2 \bar{\varsigma}+\bar{\omega}_{i}\right)\left(2-\bar{\alpha}_{i}\right)}{2\left(1+\rho_{1}^{-1}+\rho_{2}\right)}\left(\hat{x}_{i}^{k+1 \mid k}\right)^{T}\left(\Phi_{i}^{k+1 \mid k}\right)^{-1} \\
& \times \hat{x}_{i}^{k+1 \mid k}+\sum_{i=1}^{N} \sum_{j=1}^{N} \varsigma_{j}\left(\left(1+2 N \varsigma_{j}+N \omega_{i j}+2 \bar{\alpha}_{i}\right)\right. \\
& \left.+\frac{\left(1-\bar{\alpha}_{i}\right)\left(3+2 N \varsigma_{j}+N \omega_{i j}\right)}{2\left(1+\rho_{1}^{-1}+\rho_{2}\right)}\right)\left(\hat{x}_{j}^{k \mid k}\right)^{T} \Gamma^{T}\left(\Phi_{i}^{k+1 \mid k}\right)^{-1} \Gamma \\
& \times \hat{x}_{j}^{k \mid k}+\sum_{i=1}^{N} \bar{\alpha}_{i}\left(2+\left(1+2 \bar{\varsigma}+\bar{\omega}_{i}\right)\left(2-\bar{\alpha}_{i}\right)\right) \xi_{k+1}^{T} \mathcal{K}_{i}^{k+1} \\
& \times \xi_{k+1}+\sum_{i=1}^{N}\left(\varpi_{i}^{k}\right)^{T}\left(D_{i}^{k}\right)^{T} \mathcal{G}_{i}^{k+1} D_{i}^{k} \varpi_{i}^{k} \\
& +\sum_{i=1}^{N}\left(1-\bar{\alpha}_{i}\right)\left(v_{i}^{k+1}\right)^{T} \mathcal{K}_{i}^{k+1} v_{i}^{k+1} \\
& \leq \sum_{i=1}^{N} p_{i}\left(e_{i}^{k \mid k}\right)^{T}\left(\Phi_{i}^{k \mid k}\right)^{-1} e_{i}^{k \mid k}+\sum_{i=1}^{N} \frac{2+2 \bar{\varsigma}+\bar{\omega}_{i}}{1+\rho_{1}^{-1}+\rho_{2}}\left(\hat{x}_{i}^{k+1 \mid k}\right)^{T} \\
& \times\left(\Phi_{i}^{k+1 \mid k}\right)^{-1} \hat{x}_{i}^{k+1 \mid k} \\
& +\sum_{i=1}^{N} \sum_{j=1}^{N} 2 \varsigma_{j}\left(2+2 N \varsigma_{j}+N \omega_{i j}+\bar{\alpha}_{i}\right) \\
& \times\left(\hat{x}_{j}^{k \mid k}\right)^{T} \Gamma^{T}\left(\Phi_{i}^{k+1 \mid k}\right)^{-1} \Gamma \hat{x}_{j}^{k \mid k}+\sum_{i=1}^{N} 2 \bar{\alpha}_{i}\left(2+2 \bar{\varsigma}+\bar{\omega}_{i}\right) \\
& \times \xi_{k+1}^{T} \mathcal{K}_{i}^{k+1} \xi_{k+1}+\sum_{i=1}^{N}\left(\varpi_{i}^{k}\right)^{T}\left(D_{i}^{k}\right)^{T}\left(\left(\Phi_{i}^{k+1 \mid k}\right)^{-1}\right. \\
& \left.+\bar{\alpha}_{i}\left(1-\bar{\alpha}_{i}\right)\left(G_{i}^{k+1}\right)^{T} \mathcal{K}_{i}^{k+1} G_{i}^{k+1}\right) D_{i}^{k} \varpi_{i}^{k} \\
& +\sum_{i=1}^{N}\left(1-\bar{\alpha}_{i}\right)\left(v_{i}^{k+1}\right)^{T} \mathcal{K}_{i}^{k+1} v_{i}^{k+1}
\end{aligned}
$$

where

$$
\begin{aligned}
p_{i} \triangleq & \frac{1}{\lambda_{1} \bar{\omega}_{i} \underline{f}_{i}^{2}}\left(\underline{f}_{i}^{2}\left(5+3 \bar{\varsigma}+2 \bar{\omega}_{i}\right)+N \bar{f}_{i}^{2} \bar{\gamma}^{2}\left(\bar{\varsigma}+\bar{\omega}_{i}\right)\right. \\
& \left.\times\left(5+3 N \bar{\varsigma}+2 N \bar{\omega}_{i}\right)\right) .
\end{aligned}
$$

Moreover, according to (44) and Assumption 1, we know that $\left(\Phi_{i}^{k+1 \mid k}\right)^{-1}$ and $\mathcal{K}_{i}^{k+1}$ are both bounded. In consideration of the boundedness of $\hat{x}_{i}^{k+1 \mid k}$ and $\hat{x}_{i}^{k \mid k}$, we conclude that the sum of the last five terms on the right-hand side of (63) is bounded, i.e., there exists a positive scalar $t_{0}$ such that

$$
\mathbb{E}\left\{V_{k+1}\left(e^{k+1 \mid k+1}\right) \mid e^{k \mid k}\right\} \leq \sum_{i=1}^{N} p_{i}\left(e_{i}^{k \mid k}\right)^{T}\left(\Phi_{i}^{k \mid k}\right)^{-1} e_{i}^{k \mid k}+t_{0} .
$$

To this end, it follows from (45), (64), (65) and Lemma 6 that $e_{i}^{k \mid k}$ has the EMSB under conditions (54).

Remark 3: In this paper, the RF problem has been addressed for state-saturated CNs with UCSs and deception 
This article has been accepted for publication in a future issue of this journal, but has not been fully edited. Content may change prior to final publication. Citation information: DOI 10.1109/TCYB.2021.3067822, IEEE Transactions on Cybernetics

SUBMITTED

attacks, and the RF algorithm has been designed by the aid of the mathematical induction method. Note that our approach only uses the local and neighboring information to compute the gain for each node. In Theorem 2, a novel RF algorithm has been presented by finding upper bounds on error covariances. In Theorem 3, sufficient conditions have been acquired to guarantee the exponential boundedness of our RF scheme. At last, sufficient conditions have been established to ensure the EMSB of filtering errors $e_{i}^{k \mid k}$.

Remark 4: The paper solves the RF issue for CNs with state saturations and UCSs suffering from deception attacks. The primary features of our approach are highlighted as follows: 1) the state saturations, the UCSs, and the deception attacks are simultaneously considered in a unified framework; and 2) the EMSB of filtering errors is analyzed. Thus, the RF scheme developed would have not only theoretical importance but also practical significance.

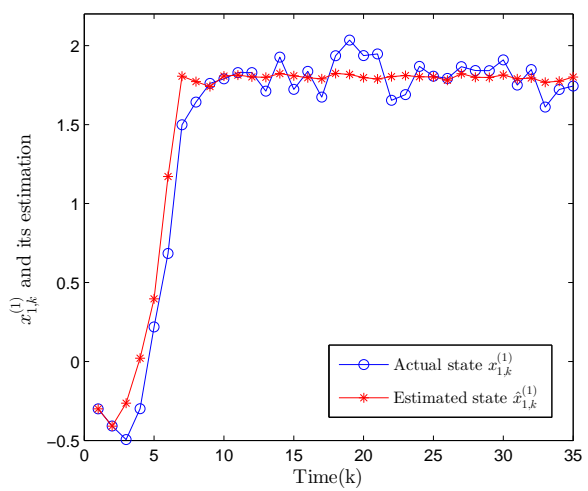

(a) $x_{1, k}^{(1)}$ and its estimate $\hat{x}_{1, k}^{(1)}$.

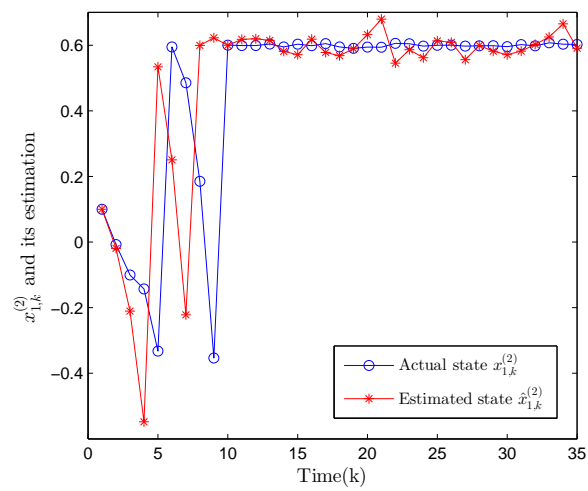

(b) $x_{1, k}^{(2)}$ and its estimate.

Fig. 1: $x_{1, k}$ and its estimates.

\section{NUMERICAL EXAMPLE}

Example 1: Consider model (1) with parameters given as follows:

$$
f\left(x_{i}^{k}\right)=\left[\begin{array}{c}
\left(x_{i}^{k}\right)^{(1)}+\sin \left(\left(x_{i}^{k}\right)^{(1)}\left(x_{i}^{k}\right)^{(2)}\right) \\
0.5\left(x_{i}^{k}\right)^{(2)}+\sin \left(\left(x_{i}^{k}\right)^{(1)}\left(x_{i}^{k}\right)^{(2)}\right)
\end{array}\right],
$$

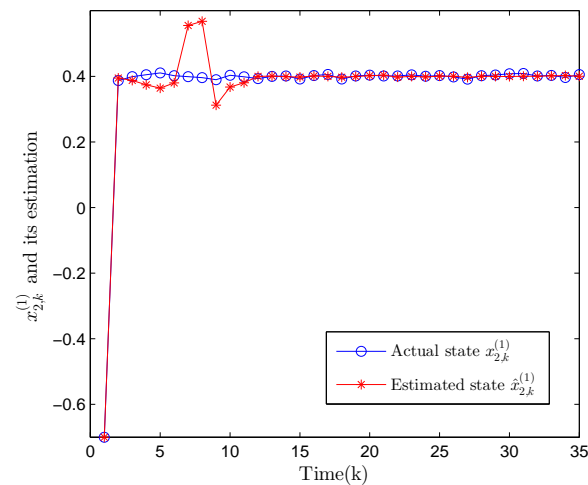

(a) $x_{2, k}^{(1)}$ and its estimate.

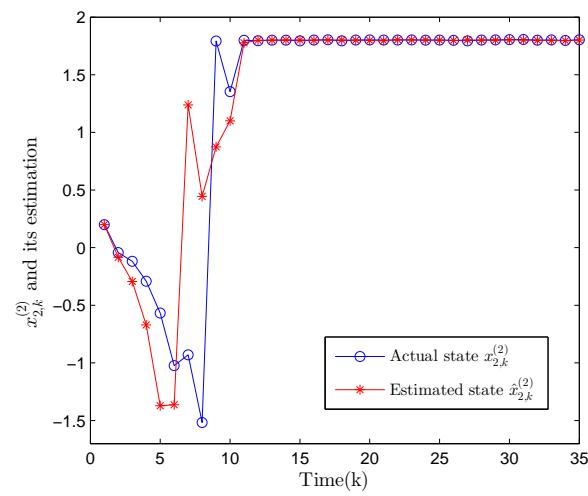

(b) $x_{2, k}^{(2)}$ and its estimate.

Fig. 2: $x_{2, k}$ and its estimate.

$$
\Omega=\left[\begin{array}{llll}
0.2 & 0.7 & 0.5 & 0.5 \\
0.8 & 0.7 & 0.4 & 0.6 \\
0.6 & 0.5 & 0.7 & 0.3 \\
0.4 & 0.8 & 0.6 & 0.7
\end{array}\right] .
$$

The upper bound of the uncertain term $\Delta \omega_{i j}$ is $\varsigma_{j}=$ $\left[\begin{array}{llll}0.1 & 0.2 & 0.2 & 0.1\end{array}\right]^{T}(j=1,2,3,4)$.

$$
\begin{aligned}
& R_{1 v}^{k}=\left[\begin{array}{cc}
0.3 & 0 \\
0 & 0.1
\end{array}\right], R_{2 v}^{k}=\left[\begin{array}{cc}
0.2 & 0 \\
0 & 0.3
\end{array}\right], \\
& R_{3 v}^{k}=\left[\begin{array}{cc}
0.4 & 0 \\
0 & 0.1
\end{array}\right], R_{4 v}^{k}=\left[\begin{array}{cc}
0.6 & 0 \\
0 & 0.2
\end{array}\right], \\
& \Gamma=\operatorname{diag}\{0.5,0.5\}, R_{i \varpi}^{k}=\operatorname{diag}\{0.5,0.3,0.4,0.2\}, \\
& G_{1}^{k}=\left[\begin{array}{cc}
0.6 & 0.25 \\
0.3 & 0.2
\end{array}\right], G_{2}^{k}=\left[\begin{array}{cc}
0.5 & 0.6 \\
0.5 & 0.2
\end{array}\right], \\
& G_{3}^{k}=\left[\begin{array}{cc}
0.8 & -1.2 \\
0.6 & 0.8
\end{array}\right], G_{4}^{k}=\left[\begin{array}{cc}
0.85 & 0.95 \\
0.5 & 0.3
\end{array}\right], \\
& D_{1}^{k}=\left[\begin{array}{c}
-0.5 \\
0.02
\end{array}\right], D_{2}^{k}=\left[\begin{array}{c}
-0.03 \\
-0.02
\end{array}\right], \\
& D_{3}^{k}=\left[\begin{array}{c}
0.02 \\
-0.6
\end{array}\right], D_{4}^{k}=\left[\begin{array}{l}
-0.4 \\
0.01
\end{array}\right] .
\end{aligned}
$$

The initial state values are $x_{1}^{0}=\left[\begin{array}{ll}-0.3 & 0.1\end{array}\right]^{T}, x_{2}^{0}=$ $\left[\begin{array}{ll}-0.7 & 0.2\end{array}\right]^{T}, x_{3}^{0}=\left[\begin{array}{ll}0.5 & -0.2\end{array}\right]^{T}$ and $x_{4}^{0}=\left[\begin{array}{ll}0.3 & -0.25\end{array}\right]^{T}$. Suppose that the saturation levels are $\pi_{1}^{\max }=\left[\begin{array}{ll}1.8 & 0.6\end{array}\right]^{T}$, $\pi_{2}^{\max }=\left[\begin{array}{ll}0.4 & 1.8\end{array}\right]^{T}, \pi_{3}^{\max }=\left[\begin{array}{ll}1.3 & 0.9\end{array}\right]^{T}$ and $\pi_{4}^{\max }=$ 
This article has been accepted for publication in a future issue of this journal, but has not been fully edited. Content may change prior to final publication. Citation information: DOI 10.1109/TCYB.2021.3067822, IEEE Transactions on Cybernetics

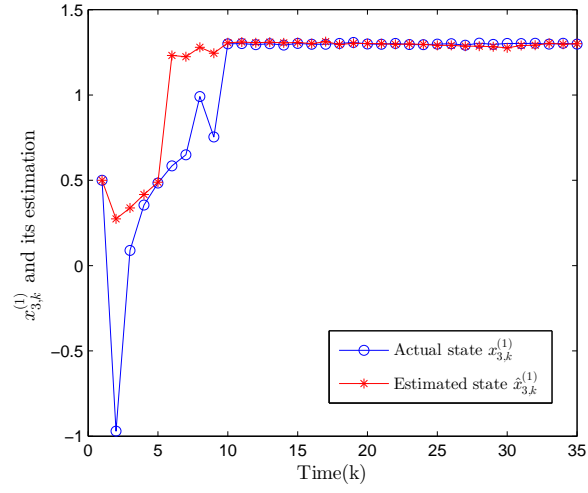

(a) $x_{3, k}^{(1)}$ and its estimate.

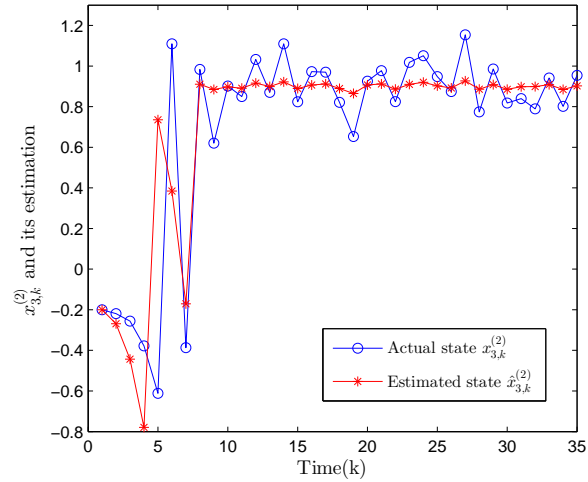

(b) $x_{3, k}^{(2)}$ and its estimate.

Fig. 3: $x_{3, k}$ and its estimate.

$\left[\begin{array}{ll}0.9 & 3.5\end{array}\right]^{T}$. Choose the positive scalars $\rho_{3}=0.8, \eta=0.9$, $\theta=0.2, \bar{\alpha}_{i}=0.6(i=1,2,3,4)$. Other parameters are selected as $\rho_{1}=10, \rho_{2}=30$ and $\lambda_{1}=280$ according to Theorem 3.

Figs. 1-4 illustrate the state and their estimation curves for four nodes, which show that the developed filters have achieved the desired performance for the state-saturated $\mathrm{CNs}$ subject to deception attacks.

Example 2: In practical application, the states of the robot are constrained from the position and orientation [11]. To explain the practicability of our scheme, consider the indoor localization problem for mobile robots networks [3], which are described by

$$
\left\{\begin{array}{c}
{\left[\begin{array}{c}
x_{i}^{k+1} \\
\kappa_{i}^{k+1} \\
\psi_{i}^{k+1}
\end{array}\right]=} \\
\sigma_{i}\left(\left[\begin{array}{c}
x_{i}^{k}+W_{i}^{k} \cos \psi_{i}^{k} \\
\kappa_{i}^{k}+W_{i}^{k} \sin \psi_{i}^{k} \\
\psi_{i}^{k}+\tau_{i}^{k}
\end{array}\right]+\sum_{j=1}^{4}\left(\omega_{i j}\right.\right. \\
\left.\left.+\Delta \omega_{i j}\right)\left[\begin{array}{c}
x_{j}^{k} \\
\kappa_{j}^{k} \\
\psi_{j}^{k}
\end{array}\right]\right)+\left[\begin{array}{c}
\left(\varpi_{i}^{k}\right)^{x} \\
\left(\varpi_{i}^{k}\right)^{\kappa} \\
\left(\varpi_{i}^{k}\right)^{\psi}
\end{array}\right] \\
y_{i}^{k}=\left[\begin{array}{lll}
1 & 0 & 0 \\
0 & 1 & 0
\end{array}\right]\left[\begin{array}{c}
x_{i}^{k} \\
\kappa_{i}^{k} \\
\psi_{i}^{k}
\end{array}\right]+v_{i}^{k}
\end{array}\right.
$$

where $\left(x_{i}^{k}, \kappa_{i}^{k}\right)$ and $\psi_{i}^{k}$ are the position and the orientation of the $i$-th robot, respectively. $\left(W_{i}^{k}, \tau_{i}^{k}\right)$ is the velocity

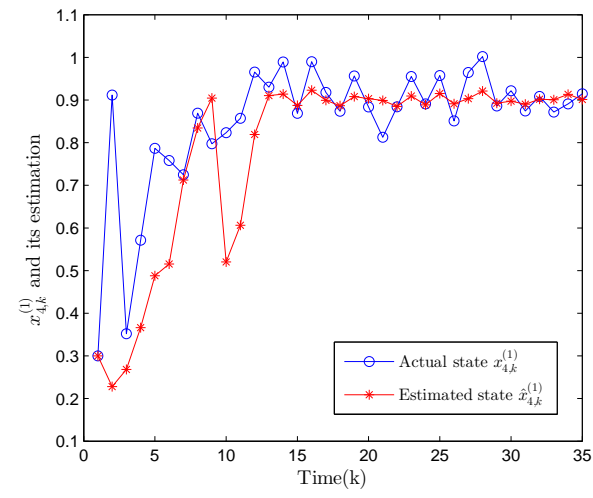

(a) $x_{4, k}^{(1)}$ and its estimate.

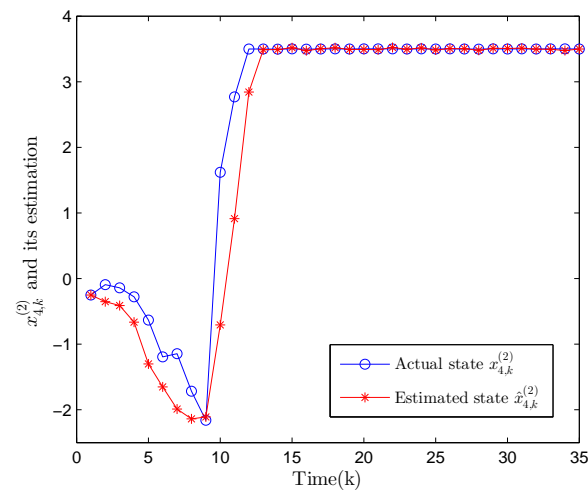

(b) $x_{4, k}^{(2)}$ and its estimate.

Fig. 4: $x_{4, k}$ and its estimate.

vector. The saturation levels are $\pi_{1}^{\max }=\left[\begin{array}{lll}13.8 & 4.6 & 11.1\end{array}\right]^{T}$, $\pi_{2}^{\max }=\left[\begin{array}{lll}9.4 & 15.8 & 7.4\end{array}\right]^{T}, \pi_{3}^{\max }=\left[\begin{array}{lll}5.3 & 6.9 & 12.8\end{array}\right]^{T}$ and $\pi_{4}^{\max }=\left[\begin{array}{lll}7.9 & 5.5 & 15\end{array}\right]^{T} \cdot \varpi_{i}^{k}=\left[\begin{array}{lll}\left(\varpi_{i}^{k}\right)^{x} & \left(\varpi_{i}^{k}\right)^{\kappa}\left(\varpi_{i}^{k}\right)^{\psi}\end{array}\right]^{T}$, $R_{i \varpi}^{k}=\operatorname{diag}\{0.3,0.3,0.3,0.3\}$ and $R_{i v}^{k}=\operatorname{diag}\{0.2,0.2\}$. The initial state values are $x_{1}^{0}=\left[\begin{array}{lll}-0.3 & 0.1 & 0.2\end{array}\right]^{T}, x_{2}^{0}=$ $\left[\begin{array}{lll}-0.7 & 0.2 & -0.1\end{array}\right]^{T}, x_{3}^{0}=\left[\begin{array}{lll}0.5 & -0.2 & 0.3\end{array}\right]^{T}$ and $x_{4}^{0}=$ $\left[\begin{array}{lll}0.3 & -0.25 & 0.1\end{array}\right]^{T}$. Other parameters are selected as the same as those in Example 1.

Fig. 5 shows the root-mean-square error (RMSE) curves in position of four robots, where Fig. 5(a) and Fig. 5(b) are the simulation results with and without taking into account the state saturation, respectively. From Fig. 5(b), one observes that the RMSE is unstable when the state saturation is not considered, which illustrates the negative impact of the state saturation on the filtering performance. Considering Fig. 5(a) with Fig. 5(b), it is clearly observed that, even under deception attacks, the performance has been greatly improved when state saturations are considered during design process. This verifies the practicability of our filter.

\section{CONClusions}

This paper has coped with the state-saturated RF issue for CNs with UCSs under deception attacks. Upper bounds on filtering errors have been acquired and filter gains have been determined through minimizing traces of these bounds. Subsequently, the EMSB of filtering errors has been analyzed. At 


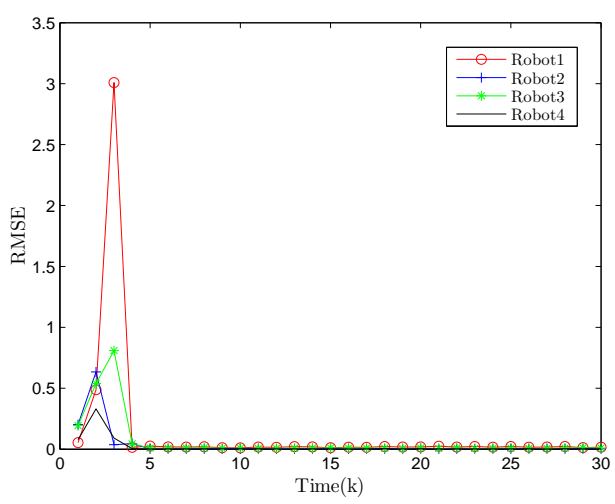

(a) RMSE in position for filter when considering state saturations.

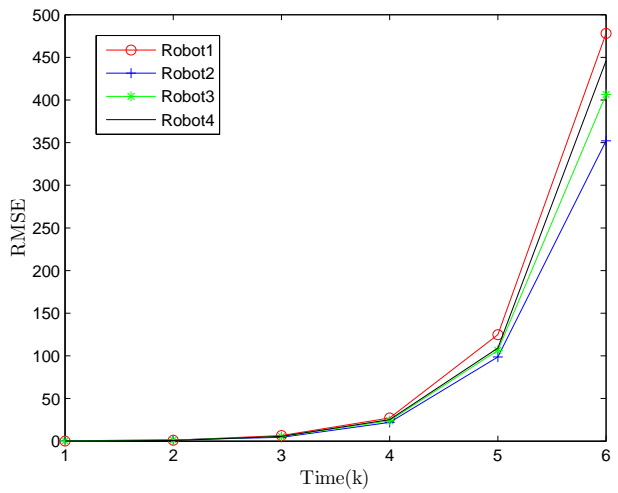

(b) RMSE in position for filter without considering state saturations.

Fig. 5: RMSE in position.

last, simulations have been provided to illustrate the usefulness of the developed RF algorithm. Future research directions will involve the RF issue for more complex systems under various network scheduling protocols, the consensus problem for discrete-time CNs, the fault estimation problem based on various communication mechanisms and the control issue for time-varying CNs [9], [12], [13], [16], [25], [46], [49], [51].

\section{REFERENCES}

[1] M. V. Basin, A. G. Loukianov and M. Hernandez-Gonzalez, Joint state and parameter estimation for uncertain stochastic nonlinear polynomial systems, International Journal of Systems Science, vol. 44, no. 7, pp. 1200-1208, 2013.

[2] R. Caballero-Águila, A. Hermoso-Garazo and J. Linares-Pérez, Distributed fusion filters from uncertain measured outputs in sensor networks with random packet losses, Information Fusion, vol. 34, pp. 7079, 2017.

[3] X. Chen and Y. Jia, Indoor localization for mobile robots using lampshade corners as landmarks:visual system calibration, feature extraction and experiments, International Journal of Control Automation \& Systems, vol. 12, no. 6, pp. 1313-1322, 2014.

[4] D. Ciuonzo, A. Aubry, and V. Carotenuto, Rician MIMO channel- and jamming-aware decision fusion, IEEE Transactions on Signal Processing, vol. 65, no. 15, pp. 3866-3880, 2017.

[5] Y. Cui, Y. Liu, W. Zhang, T. Hayat and A. Alsaedi, Sampled-data state estimation for a class of delayed complex networks via intermittent transmission, Neurocomputing, vol. 260, pp. 211-220, 2017.
[6] D. Ding, Z. Wang, D. W. C. Ho and G. Wei, Distributed recursive filtering for stochastic systems under uniform quantizations and deception attacks through sensor networks, Automatica, vol. 78, pp. 231-240, 2017.

[7] D. Ding, G. Wei, S. Zhang and Y. Liu, On scheduling of deception attacks for discrete-time networked systems equipped with attack detectors, Neurocomputing, vol. 219, pp. 99-106, 2017.

[8] H. Gao, H. Dong, Z. Wang and F. Han, An event-triggering approach to recursive filtering for complex networks with state saturations and random coupling strengths, IEEE Transactions on Neural Networks and Learning Systems, DOI: 10.1109/TNNLS.2019.2953649.

[9] X. Ge, Q.-L. Han and Z. Wang, A dynamic event-triggered transmission scheme for distributed set-membership estimation over wireless sensor networks, IEEE Transactions on Cybernetics, vol. 49, no. 1, pp. 171$183,2019$.

[10] F. Han, G. Wei, D. Ding and Y. Song, Finite-horizon bounded $H_{\infty}$ synchronisation and state estimation for discrete-time complex networks: local performance analysis, IET Control Theory and Applications, vol. 11 , no. 6 , pp. 827-837, 2017.

[11] W. He and Y. Dong, Adaptive fuzzy neural network control for a constrained robot using impedance learning, IEEE Transactions on Neural Networks and Learning Systems, vol. 29, no. 4, pp. 1174-1186, 2018.

[12] C. Huang, B. Shen, H. Chen and H. Shu, A dynamically eventtriggered approach to recursive filtering with censored measurements and parameter uncertainties, Journal of the Franklin Institute, vol. 356, no. 15 , pp. $8870-8889,2019$.

[13] C. Jia, J. Hu, D. Chen, Y. Liu and F. E. Alsaadi, Event-triggered resilient filtering with stochastic uncertainties and successive packet dropouts via variance-constrained approach, International Journal of General Systems, vol. 47, no. 5, pp. 1-16, 2018.

[14] X. Jin and G. Yang, Adaptive synchronization of a class of uncertain complex networks against network deterioration, IEEE Transactions on Circuits and Systems I Regular Papers, vol. 58, no. 6, pp. 1396-1409, 2011.

[15] X. Kai, C. Wei and L. Liu, Robust extended Kalman filtering for nonlinear systems with stochastic uncertainties, IEEE Transactions on Systems, Man, and Cybernetics - Part A: Systems and Humans, vol. 40, no. 2, pp. 399-405, 2010.

[16] H. R. Karimi, Robust $H_{\infty}$ filter design for uncertain linear systems over network with network-induced delays and output quantization, Modeling Identification and Control, vol. 30, no. 1, pp. 27-37, 2009.

[17] C. Kwon and I. Hwang, Reachability analysis for safety assurance of cyber-physical systems against cyber attacks, IEEE Transactions on Automatic Control, vol. 63, no. 7, pp. 2272-2279, 2018.

[18] Q. Li, B. Shen, J. Liang, and H. Shu, Event-triggered synchronization control for complex networks with uncertain inner coupling, International Journal of General Systems, vol. 44, no. 2, pp. 212-225, 2015.

[19] Q. Li, B. Shen, Z. Wang and F. E. Alsaadi. Event-triggered $H_{\infty}$ state estimation for state-saturated complex networks subject to quantization effects and distributed delays, Journal of the Franklin Institute, vol. 355, no. 5, pp. 2874-2891, 2018.

[20] W. Li, Y. Jia and J. Du, Recursive state estimation for complex networks with random coupling strength, Neurocomputing, vol. 219 , pp. 1-8, 2017.

[21] W. Li, Y. Jia and J. Du, State estimation for stochastic complex networks with switching topology, IEEE Transactions on Automatic Control, vol. 62, no. 12, pp. 6377-6384, 2017.

[22] W. Li, Y. Jia and J. Du, Variance-constrained state estimation for nonlinearly coupled complex networks, IEEE Transactions on Cybernetics, vol. 48, no. 2, pp. 818-824, 2017.

[23] W. Li, C. Meng, Y. Jia and J. Du, Recursive filtering for complex networks using non-linearly coupled UKF, IET Control Theory and Applications, vol. 12, no. 4, pp. 549-555, 2017.

[24] W. Li, J. Sun, Y. Jia, J. Du and X. Fu, Variance-constrained state estimation for nonlinear complex networks with uncertain coupling strength, Digital Signal Processing, vol. 67, pp. 107-115, 2017.

[25] X. Li, F. Han, N. Hou, H. Dong and H. Liu, Set-membership filtering for piecewise linear systems with censored measurements under RoundRobin protocol, International Journal of Systems Science, vol. 51, no. 9 , pp. 1578-1588, DOI: 10.1080/00207721.2020.1768453.

[26] D. Liu, Z. Wu and Q. Ye, Adaptive synchronization of an uncertain coupling complex network with time-delay, Nonlinear Dynamics, vol. 77, no. 3, pp. 643-653, 2014.

[27] J. Liu and F. Wu, State estimation for complex network systems with quantization and event-triggered communication scheme, The 35th Chi- 
This article has been accepted for publication in a future issue of this journal, but has not been fully edited. Content may change prior to final publication. Citation information: DOI 10.1109/TCYB.2021.3067822, IEEE Transactions on Cybernetics

nese Control Conference, Chengdu, China, 27-29 Jul. 2016, pp. 74347439, 2016.

[28] S. Liu, Schur Complement, Encyclopedia of statistical sciences, John Wiley \& Sons, Inc., USA: NY, 2008.

[29] X. Liu, D. W. C. Ho and C. Xie, Prespecified-time cluster synchronization of complex networks via a smooth control approach, IEEE Transactions on Cybernetics, vol. 50, no. 4, pp. 1771-1775, Apr. 2020.

[30] R. Lu, S. Chen, Y. Xu, H. Peng and K. Xie, State estimation for complex networks with randomly varying nonlinearities and sensor failures, Complexity, vol. 21, no. S2, pp. 507-517, 2016.

[31] W. Lu and T. Chen, Global synchronization of discrete-time dynamical network with a directed graph, IEEE Transactions on Circuits and Systems II: Express Briefs, vol. 54, no. 2, pp. 136-140, 2007.

[32] L. Ma, Z. Wang, Q.-L. Han and H. K. Lam, Variance-constrained distributed filtering for time-varying systems with multiplicative noises and deception attacks over sensor networks, IEEE Sensors Journal, vol. 17, no. 7, pp. 2279-2288, 2017.

[33] R. Nie, Z. Sun and J. Wang, Adaptive synchronization between two complex networks with random coupling strength, Application Research of Computers, vol. 31, no. 4, pp. 5-10, 2014.

[34] S. K. Patra and A. Ghosh, Statistics of Lyapunov exponent spectrum in randomly coupled kuramoto oscillators, Physical Review E, vol. 93 , no. 3, pp. 2208-2213, 2016.

[35] H. Peng, R. Lu, Y. Xu and F. Yao, Dissipative non-fragile state estimation for Markovian complex networks with coupling transmission delays, Neurocomputing, vol. 275, pp. 1576-1584, 2018.

[36] H. Peng, R. Lu, Y. Xu and F. Yao, Dissipative non-fragile state estimation for Markovian complex networks with coupling transmission delays, Neurocomputing, vol. 275, pp. 1576-1584, 2018.

[37] K. Reif, S. Gunther, E. Yaz and R. Unbehauen, Stochastic stability of the discrete-time extended Kalman filter, IEEE Transactions on Automatic Control, vol. 44, no. 4, pp. 714-728, 1999.

[38] P. S. Skardal, D. Taylor and J. Sun, Optimal synchronization of directed complex networks, Chaos, vol. 26, no. 9, pp. 93, 2016.

[39] A. Shirin, I. Klickstein and F. Sorrentino, Optimal control of complex networks: Balancing accuracy and energy of the control action, Chaos, vol. 27 , no. 4, pp. 1-9, 2017.

[40] R. Sasirekha and R. Rakkiyappan, Extended dissipativity state estimation for switched discrete-time complex dynamical networks with multiple communication channels: A sojourn probability dependent approach, Neurocomputing, vol. 267, pp. 55-68, 2017.

[41] L. Shi, H. Zhu, S. Zhong, K. Shi and J. Cheng. Cluster synchronization of linearly coupled complex networks via linear and adaptive feedback pinning controls, Nonlinear Dynamics, vol. 88, no. 2, pp. 859-870, 2017.

[42] Y. Theodor and U. Shaked, Robust discrete-time minimum-variance filtering, IEEE Transactions on Signal Processing, vol. 44, no. 2, pp. 181-189, 1996.

[43] D. Wang, Z. Wang, B. Shen and F. E. Alsaadi, Security-guaranteed filtering for discrete-time stochastic delayed systems with randomly occurring sensor saturations and deception attacks, International Journal of Robust and Nonlinear Control, vol. 27, no. 7, pp. 1194-1208, 2017.

[44] F. Wang, J. Liang, Z. Wang and F. E. Alsaadi, Robust synchronization of complex networks with uncertain couplings and incomplete information, International Journal of General Systems, vol. 45, no. 5, pp. 1-15, 2016.

[45] X. Wang, G. Jiang and X. Wu, State estimation for general complex dynamical networks with incompletely measured information, Entropy, vol. 20, no. 1, 2018.

[46] Y. Wu, B. Jiang and N. Lu, A Descriptor System Approach for Estimation of Incipient Faults With Application to High-Speed Railway Traction Devices, IEEE Transactions on Systems, Man, and Cybernetics: Systems, vol. 49, no. 10, pp. 2108-2118, 2019.

[47] Z. Wu, Z. Xu, P. Shi, M. Z. Chen and H. Su, Nonfragile state estimation of quantized complex networks with switching topologies, IEEE Transactions on Neural Networks and Learning Systems, vol. 29, no. 10, pp. 5111-5121, 2018.

[48] Y. Xu, R. Lu, H. Peng, K. Xie and A. Xue, Asynchronous dissipative state estimation for stochastic complex networks with quantized jumping coupling and uncertain measurements, IEEE Transactions on Neural Networks and Learning Systems, vol. 28, no. 2, pp. 268-277, 2017.

[49] W. Xu, Z. Wang and D. W. C. Ho, Finite-horizon $H_{\infty}$ consensus for multiagent systems with redundant channels via an observer-type eventtriggered scheme, IEEE Transactions on Cybernetics, vol. 48, no. 5, pp. 1567-1576, 2017.

[50] X. Yang, J. Lam, D. W. C. Ho and Z. Feng, Fixed-time synchronization of complex networks with impulsive effects via nonchattering control, IEEE Transactions on Automatic Control, vol. 62, no. 11, pp. 5511$5521,2017$.
[51] X.-M. Zhang, Q.-L. Han, X. Ge, D. Ding, L. Ding, D. Yue and C. Peng, Networked control systems: A survey of trends and techniques, IEEECAA Journal of Automatica Sinica, vol. 7, no. 1, pp. 1-17, 2020. 